\title{
Laws of Black Hole Mechanics from Holst Action
}

\author{
Ayan Chatterjee* and Amit Ghosh ${ }^{\dagger}$ \\ Theory Division, Saha Institute of Nuclear Physics, Kolkata 700064, India
}

\begin{abstract}
The formulation of Weak Isolated Horizons (WIH) based on the Isolated Horizon formulation of black hole horizons is reconsidered. The first part of the paper deals with the derivation of laws of mechanics of a WIH. While the zeroth law follows from the WIH boundary conditions, first law depends on the action chosen. We construct the covariant phase space for a spacetime having an WIH as inner boundary for the Holst action. This requires the introduction of new potential functions so that the symplectic structure is foliation independent. We show that a precise cancellation among various terms leads to the usual first law for WIH. Subsequently, we show from the same covariant phase space that for spherical horizons, the topological theory on the inner boundary is a $U(1)$ Chern-Simons theory.
\end{abstract}

PACS numbers: 04070B, 0420

\section{INTRODUCTION}

In general relativity black holes are exact solutions of Einstein's equations and are the simplest macroscopic objects of Nature [1]. Therefore, it is only natural that such objects are perfect laboratories for the search of the quantum theory of general relativity (much like the way solitons are ideal laboratories of any non-perturbative quantum theory of non-abelian gauge fields interacting with scalar fields). In the last century one of the major development in the study of black hole physics came through the realization that these solutions are analogous to some macrostates in thermal equilibrium. Dynamical processes involving only black holes obey laws that are qualitatively similar to the four laws of thermodynamics - the zeroth, first, second and the third laws. The proofs of these laws, called the laws of black hole dynamics, were first given by [2], where black hole spacetimes were supposed to contain some event horizons. However, it was soon realized that the notion of event horizons is too impractical. The global features of such horizons (for example one needs the entire asymptotic future null infinity to know whether an event horizon is present) percolate everywhere in the derivation of the said four laws of black hole dynamics [2], making the laws too abstract and unsuitable for use in many practical situations. notion of event horizons (see [3] for details). Killing horizons, which were introduced as a practical alternative to the event horizons, are closer to reality. First of all, Killing horizons are almost local, requiring the Killing vector only on and in the vicinity of the black hole horizon. The laws of black hole mechanics were also proved in this setting $[4,5]$, although it had difficulties in handling the extremal black holes at the same footing of the non-extremal ones. One difficulty being that the proofs for the laws of Killing horizon dynamics crucially depends on the existence of a bifurcating two-sphere at the horizon, which are absent for the extremal black holes. The precise dependence on the bifurcation two-sphere goes as follows: For non-extremal horizons the zeroth law states that the the surface gravity is non-zero and constant on the Killing horizon. Such a law holds if and only if the horizon is extendible to a bifurcating horizon. Furthermore, the Noether charge coming as a surface integral over a cross-section of the horizon, which is defined to be the entropy $/ 2 \pi$ in this case, is expressed in terms of all dynamical fields, their derivatives and also on the Killing field $\chi^{a}$ and its derivatives. However, the explicit dependence on $\chi^{a}$ can be eliminated provided one uses the cross-section to be the bifurcation two-sphere. One can also eliminate the second and the higher derivatives of $\chi^{a}$ by using Killing vector identities, leaving the entropy as a function of $\chi^{a}$ and $\nabla_{a} \chi^{b}$. The contribution of the term linear in $\chi^{a}$ vanishes at the bifurcation point since the Killing vector $\chi^{a}$ vanishes at that point. Finally, at the bifurcation surface one has $\nabla_{a} \chi_{b}=\epsilon_{a b}$, where $\epsilon_{a b}$ denotes the binormal. Thus, at the bifurcation point all explicit reference to the Killing field can be eliminated from the Noether charge $Q$. Thus $Q$ evaluated at the bifurcation two-sphere defines the entropy only as a function of local geometric quantities such as the metric, the matter fields and their derivatives [4]. Using the extension of the horizon one can then evaluate the entropy as an integral over an arbitrary section of the Killing horizon rather than on the bifurcation two-sphere alone, provided the surface gravity is constant and nonvanishing somewhere on the Killing horizon.

Isolated horizon $[3,6-8]$ is another local and practical alternative to a black hole horizon whose descriptions require the existence of some marginally trapped surfaces at the horizon. Since there is no explicit reference to any Killing

*Electronic address: ayan.chatterjee@saha.ac.in

†Electronic address: amit.ghosh@saha.ac.in 
vector, isolated horizons claim to do a better job than the Killing ones (see [8-10] for a detailed comparison and the recent surveys). In fact, a large number of black hole horizons are isolated horizons but not Killing horizons. The reason for this enhancement in the space of solutions is that the boundary conditions defining an isolated horizon are weaker compared to the ones defining a Killing horizon. For example, as a consequence of the isolated horizon boundary conditions it is seen that such horizons admit a Killing vector field only on the horizon, whereas a Killing horizon requires such a Killing vector field in some neighborhood of the horizon. The isolated horizon formalism was initially formulated in terms of canonical variables leading to the canonical phase space $[3,6]$. This formulation also used to show that the effective field theory on the isolated horizon is a Chern-Simons theory [11]. The detailed calculation, extensions and other consequences are discussed in [13] This also led to the calculation of the entropy done first in [12]. The covariant formulation was discussed in [7]. The various extensions of the isolated horizons and its ramifications were discussed in a series of papers [14].

One important advantage of the isolated horizons over the Killing ones is that it can deal with the extremal solutions at the same footing of non-extremal ones [15]. This requires the standard isolated horizon boundary conditions to be weakened enough so as to contain the extremal and non-extremal horizons as part of the same phase space. The standard formulation of isolated horizons takes a rigid class of null normals (which are Killing vectors only on the horizon) where the null vector fields associated with a null surface are allowed to rescale only by a positive constant. While this is definitely a possibility, the isolated horizons may actually admit a much larger class of null vectors. The new formulation, called weak isolated horizons (WIH), proposed in [15], relaxes this rigidness and allows rescaling by a class of functions. This new rescaling opens up the possibility of extending the space of solution of an isolated horizon. The standard formulation of isolated horizons place extremal and non-extremal solutions in two distinct phase spaces, much like the way they have been treated in the Killing horizon formulations; whereas in WIH, one gets a single unified space of solutions that contain both types of solutions. This implies that the laws of mechanics of WIHs, with these improved set of boundary conditions, encompass both extremal and non-extremal solutions at one go.

In this paper, we shall derive the laws of mechanics of an WIH from Holst's action [16] from a totally covariant framework. In the framework of Loop Quantum Gravity (LQG) this action is a natural starting point than the Palatini action. From this action we construct the phase space for a solution having a WIH as an inner boundary (this means that the spacetime admits an inner boundary which, in the present case, satisfies the WIH boundary conditions). The symplectic structure for this phase space is obtained, from which we prove the first law of black hole mechanics. From this covariant symplectic structure one then finds that the effective theory at the spherically symmetric WIH is precisely a $U(1)$ Chern-Simons theory. The Chern- Simons one- form gauge field is such that it does not depend on the extremal or non- extremal nature of the horizon. This shows that the effective theory for the spherical symmetric horizons, extremal or non- extremal, is a $U(1)$ Chern-Simons theory. Correspondingly, the entropy of these horizons will again be proportional to area of the horizon. That the effective topological theory on the horizon is a $U(1)$ Chern-Simons theory was also shown in $[12,13]$. However in the present calculation, we carefully derive the laws of black hole mechanics from a completely covariant formulation taking into account the weakest possible boundary conditions for a black hole horizon and then reinforce the claims that the surface symplectic structure of the WIH is that of a Chern- Simons theory.

The plan of the paper is as follows. We first recall the boundary conditions of a Weak Isolated Horizon (WIH). Then, we derive some key consequences of these boundary conditions. For example, the boundary conditions result in the zeroth law of black hole mechanics provided we restrict the equivalence class of null normals on the WIH. These boundary conditions are equally applicable to extremal as well as non-extremal WIH. Then, using the Holst action we show that the principle of least action is well defined in presence of some appropriate boundary terms. In the next step we construct the symplectic structure in the space of solutions in which each solution contains a WIH as its inner boundary. We then derive the 1st law of black hole mechanics using this symplectic structure. We also derive the Chern-Simons symplectic structure on the horizon from this formulation.

\section{WEAK ISOLATED HORIZONS}

We now give an introduction to the idea of weak isolated horizons [15]. Let us consider $\mathcal{M}$ to be a four-manifold equipped with a metric $g_{a b}$ of signature $(-,+,+,+)$. Our notations and conventions closely follow that of $[7,15] . \Delta$ is a null hypersurface in $\mathcal{M}$ of which $\ell^{a}$ is a future directed null normal. However, if $\ell^{a}$ is a null normal, so is $\xi \ell^{a}$, where $\xi$ is any arbitrary positive function on $\Delta$. Thus, $\Delta$ naturally admits an equivalence class of null normals $\left[\xi \ell^{a}\right]$. We denote by $q_{a b} \triangleq g_{a b}$ the degenerate intrinsic metric on $\Delta$ induced by $g_{a b}$ (indices that are not explicitly intrinsic

on $\Delta$ will be pulled back and $\triangleq$ means that the equality holds only on $\Delta$ ). The tensor $q^{a b}$ will be an inverse of $q_{a b}$ if it satisfies $q^{a b} q_{a c} q_{b d} \triangleq q_{c d}$. The expansion $\theta_{(\ell)}$ of the null normal $\ell^{a}$ is then defined by $\theta_{(\ell)}=q^{a b} \nabla_{a} \ell_{b}$, where $\nabla_{a}$ is 
the covariant derivative compatible with $g_{a b}$.

The null surface $\Delta$ introduced above is an arbitrary null surface equipped with an equivalence class of null normals $\left[\xi \ell^{a}\right]$. The conditions on $\Delta$ are too general to make it resemble a black hole horizon. To enrich $\Delta$ with useful and interesting information, we need to impose some restrictions on this surface. The idea is that we endow a minimal set of conditions on the null hypersurface $\Delta$ so that it behaves as a black hole horizon. As we shall see, the zeroth law and the first law of black hole mechanics will naturally follow from these conditions. These definitions will be local and only provides a construction of black hole horizon and do not define a black hole spacetime which is a global object. However, if there is a global solution, like the Schwarzschild solution, then these conditions will be satisfied.

\section{A. The First Set of Boundary Conditions}

We shall now introduce the set of boundary conditions to be imposed on the null surface $\Delta$ so that effectively the surface behaves as a black hole horizon. The boundary conditions that are proposed here are the least number of conditions that are necessary for a generic black hole horizon. Since the null surface has an equivalence class of null normals $\left[\xi \ell^{a}\right]$ as its generators, it is natural to impose the boundary conditions on all of these null normals i.e. the boundary conditions has to hold for the entire equivalence class $\left[\xi \ell^{a}\right]$.

The null surface $\Delta$ generated by the equivalence class $\left[\xi \ell^{a}\right]$ will be called a non-expanding horizon $(\mathrm{NEH})$ in $\left(\mathcal{M}, g_{a b}\right)$ if the following conditions are satisfied [15]:

1. $\Delta$ is topologically $S^{2} \times \mathbb{R}$.

2. The expansion $\theta_{(\xi \ell)} \triangleq 0$ for any $\xi \ell^{a}$ in the equivalence class.

3. The equations of motion and energy conditions hold on the surface $\Delta$ and the vector field $-T_{b}^{a} \xi \ell^{b}$ is future directed and causal.

There are some important points to note in the boundary conditions presented here. Firstly, all boundary conditions are intrinsic to $\Delta$. This implies that to describe NEH, one needs no reference to the spacetime in the exterior. Also, the definition doe not involve Killing vectors although, as we shall see, the boundary conditions imply the presence of a Killing vector on the null surface $\Delta$. Of these boundary conditions, the first one is just a topological restriction on the horizon and has no reference to the equivalence class of null normals. The second boundary condition is the most important of the conditions. The expansion freeness is a special requirement for any isolated black hole horizon i.e it holds only for those null surfaces which are black holes. Any null surface will not satisfy this condition. For example, the Minkowski light cone does not satisfy the expansion free condition. This is because the Minkowski light cone is not a black hole horizon although it appears so for the Rindlar observers. One might be tempted to infer that the second condition implies infinite number of boundary conditions to be imposed on each of the infinite number of null normals in the equivalence class $\left[\xi \ell^{a}\right]$. This however is not true. In fact, it is enough that the expansion corresponding to any one null normal is zero. The quantities involved in the boundary conditions are such that once these conditions are satisfied by one null vector $\ell^{a}$, then these are also obeyed by every null vector in the class $\left[\xi \ell^{a}\right]$. This is true also for the expansion-free condition, since $\theta_{(\xi \ell)} \triangleq \xi \theta_{(\ell)}$. So, for all the conditions defining a NEH, it is sufficient that these are satisfied by only one normal vector field in the class $\left[\xi \ell^{a}\right]$ The third boundary condition ensures that the equation of motion of all fields are satisfied on $\Delta$. This condition only allows those fields which satisfy the dominant energy condition. This requirement also holds true for all the null normals in the equivalence class $\left[\xi \ell^{a}\right]$ if it holds true for one.

\section{B. Consequences of the Boundary Conditions}

The above boundary conditions have important consequences for the kinematical structure of the horizon. First of all, note that one can have important simplifications for the null surface $\Delta$ which are quite independent of the boundary conditions. We note them below. Since any $\ell^{a}$ in $\left[\xi \ell^{a}\right]$ is normal to $\Delta$, these are twist free. Next, because the surface is null, the normal vector is also the tangent vector. The tangent vector fields are tangent to the generators of the surface. It can be shown that these generators are geodesics or in other words, each $\xi \ell^{a}$ in $\left[\xi \ell^{a}\right]$ is geodetic, i.e.

$$
\xi \ell^{a} \nabla_{a}\left(\xi \ell^{b}\right) \triangleq \kappa_{(\xi \ell)} \xi \ell^{b}
$$

where $\kappa_{(\xi \ell)}$ is the acceleration of $\xi \ell^{a}$. It can be easily deduced from (2.1) that the acceleration varies in the equivalence class

$$
\kappa_{(\xi \ell)}=\xi \kappa_{(\ell)}+£_{\ell} \xi
$$


We shall always work with the null tetrad basis $(\ell, n, m, \bar{m})$ such that $1=-n \cdot \ell=m \cdot \bar{m}$ and all other scalar products vanish. This is specially suited for the problem since one of the null normals $\ell^{a}$ matches with one of the vectors in the equivalence class $\left[\xi \ell^{a}\right]$. The spacetime metric is then given by $g_{a b}=-2 \ell_{(a} n_{b)}+2 m_{(a} \bar{m}_{b)}$.

Given the simplification that any null normal $\xi \ell^{a}$ is twist-free and the second boundary condition that any null normal $\xi \ell^{a}$ is expansion- free, the Raychaudhuri equation becomes

$$
0 \triangleq £_{\xi \ell} \theta_{(\xi \ell)} \triangleq-\left|\sigma_{(\xi \ell)}\right|^{2}-\xi^{2} R_{a b} \ell^{a} \ell^{b}
$$

where, $\sigma_{(\xi \ell)}=m^{a} m^{b} \nabla_{a}\left(\xi \ell_{b}\right)$ is the shear of $\xi \ell^{a}$. By using the energy conditions and Einstein equations, it can be shown that both terms on the right hand side of (2.3) vanish independently on $\Delta$. Therefore every null normal $\xi \ell^{a}$ in the equivalence class is also shear-free. Again, note that if any one of the null normals $\ell^{a}$ is shear free, all the null normals in the equivalence class $\left[\xi \ell^{a}\right]$ are shear free too. In short, every null normal in the equivalence class $\left[\xi \ell^{a}\right]$ is twist- free, shear- free and expansion- free. These conditions imply that there exists an one-form $\omega_{a}^{(\ell)}$ on $\Delta$, depending on $\ell^{a}$ such that

$$
\nabla_{\underline{a}} \ell^{b} \triangleq: \omega_{a}^{(\ell)} \ell^{b}
$$

The one-form defined in (2.4) plays an important role in the whole analysis. It is also clear that since the one- form $\omega_{a}^{(\ell)}$ depends on the null normals, it varies in the class $\left[\xi \ell^{a}\right]$. The variation is

$$
\omega^{(\xi \ell)}=\omega^{(\ell)}+d \ln \xi
$$

where $d$ is the exterior derivative in $\Delta$. Since the pull-back of the one-form $\xi \ell_{a}$ is zero on $\Delta$, it follows that every $\xi \ell^{a}$ in the class is a Killing vector on $\Delta$, namely $£_{(\xi \ell)} q_{a b} \triangleq 0$. A straightforward calculation (using results of appendix (IX A)) shows that the curvature of $\omega^{(\xi \ell)}$

$$
d \omega^{(\xi \ell)} \triangleq 2\left(\operatorname{Im} \Psi_{2}\right)^{2} \epsilon,
$$

where $\operatorname{Im} \Psi_{2}=C_{a b c d} \ell^{a} m^{b} \bar{m}^{c} n^{d}$ is a complex scalar, $C_{a b c d}$ is the Weyl-tensor and ${ }^{2} \boldsymbol{\epsilon}=i m \wedge \bar{m}$ is the area two- form on the cross- sections of $\Delta$. Again from equation (2.5), since the one form $\omega^{(\xi \ell)}$ varies like a $U(1)$ gauge field, the equation (2.6) will hold true for all $\omega^{(\xi \ell)}$ corresponding to the vectors in the equivalence class. The Killing equations imply that the area two- form ${ }^{2} \boldsymbol{\epsilon}$ of the cross-section is preserved under Lie-flow of every $\xi \ell^{a}$ in the class, $£_{(\xi \ell)}{ }^{2} \boldsymbol{\epsilon}_{a b} \triangleq 0$.

\section{Weak Isolated Horizon and the Zeroth Law}

Let us recall that from the point of view of boundary conditions, all the horizons generated by the null normals in the equivalence class $\left[\xi \ell^{a}\right]$ are equivalent. In other words, the boundary conditions are oblivious to the equivalence class and cannot prefer one horizon generated by say $\ell^{a}$, over another generated by $\xi \ell^{a}$, both null vectors being in the equivalence class. Again, recall from (2.2) that the accelerations $\kappa_{(\xi \ell)}$ of the null normals $\xi \ell^{a}$ vary in the equivalence class through $\kappa_{(\xi \ell)}=\xi \kappa_{(\ell)}+£_{\ell} \xi$. The point of view here is that the accelerations $\kappa_{(\xi \ell)}$ just provides a nomenclature which can be used as tags to the various surfaces generated by the corresponding null normals in the equivalence class $\left[\xi \ell^{a}\right]$. In short, all the null surfaces labelled by say $\kappa_{\left(\xi_{1} \ell\right)}, \kappa_{\left(\xi_{2} \ell\right)}, \kappa_{\left(\xi_{3} \ell\right)}$ etc are on the equivalent footing from the point of view of boundary conditions. This exemplifies the claim that the WIH boundary conditions puts the nonextremal and extremal black hole horizons in the same footing.

An noted several times, the acceleration $\kappa_{(\xi \ell)}$ varies over the class $[\xi \ell]: \kappa_{(\xi \ell)}=\xi \kappa_{(\ell)}+£_{\ell} \xi$ and is not a constant in general. In order to obtain the zeroth law, which requires the acceleration for each normal vector in the class to be a constant, we need to restrict the NEHs further. Let us call the restricted horizon the weak isolated horizon (WIH), which is a NEH equipped with a class $\left[\xi \ell^{a}\right]$ such that

$$
£_{(\xi \ell)} \omega^{(\xi \ell)} \triangleq 0 .
$$

Let us make a few comments here: First, as in Killing horizons, we will interpret the acceleration $\kappa_{(\xi \ell)}$ as the surface gravity of $\xi \ell^{a}$. However, since a global Killing field is absent, the value of the surface gravity cannot be uniquely determined. In isolated horizon formulation it is natural to keep this freedom. In fact, as we shall see below, this freedom will enable the extremal horizon with surface gravity $\kappa=0$ and non- extremal horizons $\kappa \neq 0$ to be on the same footing. 
Second, the boundary condition (2.7), unlike the previous ones, is not a single condition. Namely, if it is obeyed by one normal vector $\ell^{a}\left(£_{\ell} \omega^{(\ell)} \triangleq 0\right)$, then it is not guaranteed that every other normal vector $\xi \ell^{a}$ in the class will obey it. This pathology is eliminated by restricting the choice of $\xi \mathrm{s}$

$$
\xi=c e^{-v \kappa_{(\ell)}}+\kappa_{(\xi \ell)} / \kappa_{(\ell)}
$$

where, $c$ is a nonzero function satisfying $£_{\ell} c=0$ and $v$ is the affine parameter such that $£_{\ell} v=1$. For the rest of the paper, we choose $c \triangleq$ constant. Having restricted ourselves to this specific class it is now easy to verify that (2.7) becomes just one condition: $£_{(\xi \ell)} \omega^{(\xi \ell)} \triangleq d\left(\xi \ell \cdot \omega^{(\xi \ell)}\right) \triangleq d \kappa_{(\xi \ell)}$ for every $\xi$ belonging to the restricted class. From now on the class of normal vectors will always follow this restriction. This class admits a $\xi=c e^{-v \kappa_{(\ell)}}$, for which $\kappa_{(\xi \ell)} \triangleq 0$ when the surface gravity $\kappa_{(\ell)}$ of $\ell^{a}$ is nonzero. For obvious reasons such an isolated horizon, characterized by a normal vector of vanishing surface gravity, will be called an extremal horizon. Thus, our class of normal vectors contains both extremal and non-extremal horizons, as opposed to the constant class of normal vectors $\left[c \ell^{a}\right]$. In other words, the WIH boundary conditions cannot differentiate between the surfaces generated by null normals in the equivalence class $\left[\xi \ell^{a}\right]$ with $\xi=c e^{-v \kappa_{(\ell)}}+\kappa_{(\xi \ell)} / \kappa_{(\ell)}$ and hence the extremal and non- extremal horizons become part of the WIH.

As already noted, WIH boundary condition (2.7) is equivalent to the zeroth law: $d \kappa_{(\xi \ell)} \triangleq 0$. Therefore, the surface gravity corresponding to each $\xi \ell^{a}$ in $\left[\xi \ell^{a}\right]$ is constant on $\Delta$, provided $\xi$ s belong to the restricted class.

\section{THE HOLST ACTION}

The Holst action ([16]) is a modification of the Palatini action where a term is added which has a property that it does not contribute to the equation of motion. Let us recall that the Palatini action is constructed out of the basic fields, the tetrads $e_{a}^{I}$ and a $S O(3,1)$ Lie algebra valued connection one form $A_{I J}$ (see [17] and [18] for details). On shell, the connection $A_{I J}$ equals the spin connection. The Legendre transformation of Palatini Lagrangian to the Hamiltonian formulation introduces second class constraints. Solution of these constraints needs some gauge fixing which essentially reduces the theory to that of the standard metric variable theory and one looses the essential advantages of the connection formulation. One can however go to the self dual complex connections where the theory is much easier but this also creates problems for the quantum theory. Indeed, the quantum theory based on LQG needs background independent analysis on the connection space. However, such a theory is still to be constructed and in the meantime, the quantization programme is successful on the phase space of the real variables. This phase space is the Barbero- Immirzi phase space constructed out of the original Palatini phase space by a one parameter canonical transformation labelled by the Barbero- Immirzi parameter $\gamma$. The Holst action is precisely the action whose Legendre transformation gives the Barbero- Immirzi phase space.

Let us first begin with the Palatini action. We consider the spacetime $\mathcal{M}$ which is bounded by the the Cauchy surfaces $M_{ \pm}$and intersecting at $i^{0}$. For now, we shall only deal with a spacetime without any inner boundary $\Delta$. The action is given by:

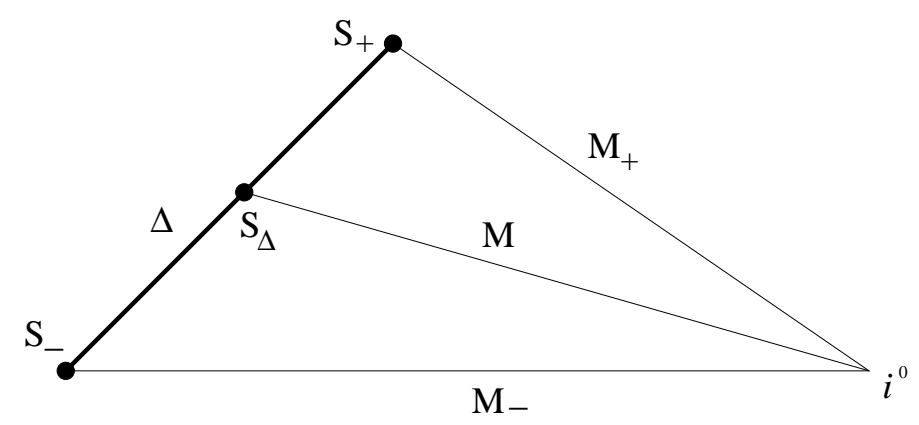

FIG. 1: $M_{ \pm}$are two partial Cauchy surfaces enclosing a region of space-time and intersecting $\Delta$ in the 2 -spheres $S_{ \pm}$respectively and extend to spatial infinity $i^{\circ}$. Another Cauchy slice M is drawn which intersects $\Delta$ in $S_{\Delta}$

$$
\begin{aligned}
S_{P} & =\frac{1}{32 \pi G} \int_{\mathcal{M}} \epsilon_{I J K L} e^{I} \wedge e^{J} \wedge F^{K L} \\
& =\frac{1}{16 \pi G} \int_{\mathcal{M}} \Sigma_{I J} \wedge F^{I J}
\end{aligned}
$$


where, $\Sigma_{I J}=\frac{1}{2} \epsilon_{I J K L} e^{K} \wedge e^{L}$ is a two- form defined for future convenience. The variation of the integral with respect to $A_{I J}$ will lead to :

$$
\delta S_{P}=\frac{1}{16 \pi G} \int_{M} d\left(\Sigma_{I J} \wedge \delta A^{I J}\right)-\frac{1}{16 \pi G} \int_{M} D \Sigma_{I J} \wedge \delta A^{I J}
$$

where we have used the fact that:

$$
D \Sigma_{I J}:=d \Sigma_{I J}+A_{I}^{K} \wedge \Sigma_{K J}+A_{J}^{K} \wedge \Sigma_{I K}
$$

Thus, the equation of motion obtained from variation of the connection is :

$$
D \Sigma_{I J}=0
$$

It is then easy to check from (3.4) that the connection $A_{I J}$ is the spin connection. Then, the variation of the action (3.1) with respect to the tetrads $e_{I}^{a}$ gives the Einstein equation. In the present case, observe that the boundary term goes to zero at the outer boundary where the spacetime is flat and the variational principle is well defined. In cases where there is an inner boundary, we need to take care that the boundary term again vanishes.

Now, let us consider the Holst action [16]:

$$
S_{H}=S_{P}-\frac{1}{16 \pi G \gamma} \int_{M} e^{I} \wedge e^{J} \wedge F_{I J}
$$

where, $\gamma$ is a real constant called the Barbero -Immirzi parameter. Note that written in this form, the parameter cannot be equal to zero. For $\gamma=-i(+i)$, we get the self dual (anti self-dual) action. Then, the action $S_{H}$ can be written in terms of ${ }^{ \pm} F_{I J}\left(A_{I J}\right)=F_{I J}\left({ }^{ \pm} A_{I J}\right)$. In these cases, it can be argued that the connection is actually a self-dual (anti-self dual) part of the spin connection. For generic but other values of $\gamma$, it is also true that the connection $A_{I J}$ is actually a spin connection, completely determined by the tetrad. The variation of the action with respect to the tetrad again reproduces the Einstein equation, the $\gamma$ - dependent term, on shell, being zero by the algebraic Bianchi identity.

The extra $\gamma$ - dependent term that appears in the action is important for quantum mechanical reasons. It is in some sense similar to the theta $(\theta)$ term in QCD. It is well known that in Yang- Mills theory, $\theta$ - term introduces inequivalent $\theta$-sectors for the corresponding quantum theory. In the similar way, the extra term here introduces the inequivalent $\gamma$-sectors for quantum general relativity [19]. However, while the $\theta\left(\mathbf{F}_{Y M^{*}} \mathbf{F}_{Y M}\right)$ is a purely topological term ${ }^{1}$, being equal to a total divergence of a 3 -form, the $\gamma$ - dependent term here vanishes because of the first Bianchi identity. One can show that the phase space corresponding to these two theories are equal. To see this one can construct the symplectic structure for the Holst action and argue that if the phase space vectors satisfy the linearized version of the spin connection equation, the $\gamma$ - dependent term disappears (see appendix IX B for the details). In other words, the Holst modification of the Palatini action implies a canonical transformation on the phase space.

\section{A. The Action and the Variational Principle}

In this subsection, we will use the Holst action for a spacetime with inner boundary. In the present case, the inner boundary is a null surface which satisfies the WIH boundary conditions. To be more precise, the spacetime under consideration is a region bounded by the Cauchy surfaces $M_{1}$ and $M_{2}$ extending to spatial infinity and the null surface $\Delta$ (see fig. 1). The variation of the fields will be between all those configurations which satisfy the boundary conditions at infinity and at $\Delta$. In particular, we consider those variations of $\left(e^{I}{ }_{a}, A_{I J}\right)$ which satisfy the standard fall off conditions at infinity and on $\Delta$, satisfy the following conditions:

1. each spacetime admits a null normal belonging to the equivalence class $\left[\xi \ell^{a}\right]$.

2. each pair $\left(\Delta,\left[\xi \ell^{a}\right]\right)$ is a WIH.

We can always introduce a fixed set of internal null vectors $\left(\ell^{I}, n^{I}, m^{I}, \bar{m}^{I}\right)$ on $\Delta$ such that $\partial_{a}\left(\ell^{I}, n^{I}, m^{I}, \bar{m}^{I}\right)=0$ (this partially fixes the Lorentz frame). Given these internal null vectors and the tetrad $e_{a}^{I}$, we can construct the null vectors $\left(\ell^{a}, n^{a}, m^{a}, \bar{m}^{a}\right)$ through $\ell_{a}=e^{I}{ }_{a} \ell_{I}$.

\footnotetext{
${ }^{1}$ In some String theories, like the $E_{8} \otimes E_{8}$ heterotic string theory, the $\theta$ - term arises from the Yang- Mills Chern-Simons term which makes the theory gauge anomaly free. In that case, derivative of $\theta$ is a field dual to the Kalb- Ramond three form. We consider the case where $\theta$ in a constant just like in ordinary QCD.
} 


\section{Tetards and Connection on $\Delta$}

To proceed further, we need the the expressions for the tetrad and the connection in terms of the null vectors. The expansion of tetrads in terms of the null vectors can be easily calculated from the expression of tetrads relating the spacetime metric $g_{a b}$ and internal flat metric $\eta_{I J}$. The expression is given by:

$$
e^{I} \underset{\leftarrow}{\leftarrow} \triangleq-n_{a} \ell^{I}+m_{a} \bar{m}^{I}+\bar{m}_{a} m^{I}
$$

The above equation (3.6) can be used to get an expression for the product of tetrads:

$$
\begin{aligned}
e_{\stackrel{a}{I}}^{I} \wedge e^{J} \underset{\leftarrow}{b} & \triangleq-2 n_{a} \wedge m_{b} \ell^{[I} \bar{m}^{J]}-2 n_{a} \wedge \bar{m}_{b} \ell^{[I} m^{J]}+2 m_{a} \wedge \bar{m}_{b} \bar{m}^{[I} m^{J]} \\
& =-2 n_{a} \wedge m_{b} \ell^{[I} \bar{m}^{J]}-2 n_{a} \wedge \bar{m}_{b} \ell^{[I} m^{J]}+2 i m^{[I} \bar{m}^{J]}{ }^{2} \boldsymbol{\epsilon}_{a b}
\end{aligned}
$$

Using this expression for the tetrad products (3.7), and the expansion for the internal epsilon tensor $\epsilon_{I J K L}=$ $4 ! \ell_{[I} n_{J} m_{K} \bar{m}_{L]}$, we get

$$
\Sigma_{a b}^{I J} \triangleq 2 \ell^{[I} n^{J] 2} \epsilon_{a b}+2 n_{a} \wedge\left(i m_{b} \ell^{[I} \bar{m}^{J]}-i \bar{m}_{b} \ell^{[I} m^{J]}\right)
$$

We are now in a position to calculate the connection $A_{I J}$. We will be using the Newman- Penrose formalism. The details of the Newman- Penrose coefficients are given in the appendix IX A. Using those expansions, one gets the following expression for the covariant derivatives of the null normals pulled back and restricted to $\Delta$.

$$
\begin{gathered}
\nabla_{a} \ell^{b} \triangleq \omega_{a}^{(\ell)} \ell^{b} \\
\nabla_{\llcorner} n^{b} \triangleq-\omega_{a}^{(\ell)} n^{b}+\bar{U}_{a}^{(l, m)} m^{b}+U_{a}^{(l, m)} \bar{m}^{b} \\
\nabla_{\leftarrow} m^{b} \triangleq U^{(l, m)}{ }_{a} \ell^{b}+V_{a}^{(m)} m^{b} \\
\nabla_{a} \bar{m}^{b} \triangleq \bar{U}_{a}^{(l, m)} \ell^{b}-V_{a}^{(m)} \bar{m}^{b}
\end{gathered}
$$

where, the superscripts for each of the one forms keep track of their dependencies on the rescaling of the corresponding null normals. The expressions of the one forms $\omega^{(\ell)}, U^{(l, m)}, \bar{U}^{(l, m)}$ and $V^{(m)}$ can be written in terms of the null normals and are as follows:

$$
\begin{aligned}
\omega_{a}^{(\ell)} & \triangleq-(\epsilon+\bar{\epsilon}) n_{a}+(\bar{\alpha}+\beta) \bar{m}_{a}+(\alpha+\bar{\beta}) m_{a} \\
U_{a}^{(l, m)} & \triangleq-\bar{\pi} n_{a}+\bar{\mu} m_{a}+\bar{\lambda} \bar{m}_{a} \\
V_{a}^{(m)} & \triangleq-(\epsilon-\bar{\epsilon}) n_{a}+(\beta-\bar{\alpha}) \bar{m}_{a}+(\alpha-\bar{\beta}) m_{a}
\end{aligned}
$$

The part of the connection $V^{(m)}$ is purely imaginary. Let us at this stage point out the result of the rescaling of the null normal $\ell^{a}$ on the various quantities of interest. Firstly, for $\ell^{a} \longrightarrow \xi \ell^{a}$, we have:

$$
\omega_{a}^{(\ell)} \rightarrow \omega_{a}^{(\xi \ell)}=\omega_{a}^{(\ell)}+\nabla_{a} \ln \xi
$$

Since the normalization of $\ell^{a}$ and $n^{a}$ are connected, we must have $n^{a} \longrightarrow \frac{n^{a}}{\xi}$ when $\ell^{a} \longrightarrow \xi \ell^{a}$. Then the effect of the rescaling can be seen to be:

$$
\nabla_{\underline{\leftarrow}}\left(\frac{n^{b}}{\xi}\right) \triangleq-\omega_{a}^{(\xi \ell)}\left(\frac{n^{b}}{\xi}\right)+\bar{U}_{a}^{(\xi \ell, m)} m^{b}+U_{a}^{(\xi \ell, m)} \bar{m}^{b}
$$

Thus, under this transformation, we have that the one- form $\omega^{(\xi \ell)}$ transforms in the usual way: $\omega^{(\ell)} \rightarrow \omega_{a}^{(\xi \ell)}=$ $\omega_{a}^{(\ell)}+\nabla_{a} \ln \xi$ and the other one forms $U^{(l, m)}$ and $\bar{U}^{(l, m)}$ transform as

$$
\begin{aligned}
\bar{U}_{a}^{(\ell, m)} \longrightarrow \bar{U}_{a}^{(\xi \ell, m)} & =\frac{\bar{U}_{a}^{(\ell, m)}}{\xi} \\
U_{a}^{(\ell, m)} \longrightarrow U_{a}^{(\xi \ell, m)} & =\frac{U_{a}^{(\ell, m)}}{\xi}
\end{aligned}
$$


This rescaling is in the sector of $\ell, n$. There can be another set of rescaling quite independent of the rescaling of $\ell, n$. This concerns the transformation in the other set of null vectors $m, \bar{m}$ of the null tetrad. This transformation function will also be independent of the function $\xi$. Now, for $m \rightarrow f m$ and for $\bar{m} \rightarrow \frac{\bar{m}}{f}$, where $f$ is any function on $\Delta$, we have the following transformations:

$$
\begin{aligned}
& \nabla_{\leftarrow}\left(f m^{b}\right) \triangleq U_{a}^{(\ell, f m)} \ell^{b}+V_{a}^{(f m)}\left(f m^{b}\right) \\
& \nabla_{\leftarrow}\left(\frac{\bar{m}}{f}\right) \triangleq \bar{U}_{a}^{(\ell, f m)} \ell^{b}-V_{a}^{(f m)}\left(\frac{\bar{m}^{b}}{f}\right)
\end{aligned}
$$

The transformation rules are as follows for the one forms $U_{a}^{(\ell, m)}, \bar{U}_{a}^{(\ell, m)}$ and $V_{a}^{(m)}$ are as follows:

$$
\begin{aligned}
\bar{U}_{a}^{(\ell, m)} & \longrightarrow \bar{U}_{a}^{(\ell, f m)}=\frac{\bar{U}_{a}^{(\ell, m)}}{f} \\
U_{a}^{(\ell, m)} & \longrightarrow U_{a}^{(\ell, f m)}=f U_{a} \\
V_{a}^{(m)} & \longrightarrow V_{a}^{(f m)}=V_{a}^{(m)}+\nabla_{a} \ln f
\end{aligned}
$$

The part of the connection $\omega^{(\ell)}$ and $V^{(m)}$ transform as $U(1)$ field whereas the the other parts of connections only rescale. We have constrained only one part of the connection while defining the Weak Isolated Horizon in the sense that only the one form $\omega$ is constrained. The other part of the connection is left as it is. If we want to constrain more of the parts of the connection then we get the definition of the Isolated horizon.

We can use these information to find the connection. To do this, we first note that the internal null vectors are fixed such that $\partial_{a}\left(\ell^{I}, n^{I}, m^{I}, \bar{m}^{I}\right)=0$. Then, we get

$$
\nabla_{\leftarrow} \ell_{I} \triangleq A_{a} I J \ell_{J}
$$

We can choose a tetrad $e_{a}^{I}$ which maps the vector $\ell_{I}$ to $\ell_{a}$. This tetrad is annihilated by the covariant derivative, $\nabla_{a} e_{b}^{I}=0$. Then the equation (3.21) gives: $A_{\leftarrow}{ }_{\leftarrow}^{I} \ell^{J} \triangleq \omega_{a}^{(\ell)} \ell^{I}$. Written is a more compact form, this reduces to:

$$
A_{\underset{a}{\leftarrow} I J} \triangleq-2 \omega_{a}^{(\ell)} \ell_{[I} n_{J]}+Q_{I J}
$$

where, the one form $Q_{I J}$ is such that $Q_{I J} \ell^{J} \triangleq 0$.

We can proceed just as before for the other null vectors $n_{I}, m_{I}$ and $\bar{m}_{I}$. For the null vector $n_{I}$, that gives us:

$$
A_{\underset{\leftarrow}{\leftarrow} I J} \triangleq-2 \omega_{a}^{(\ell)} \ell_{[I} n_{J]}-2 \bar{U}_{a}^{(\ell, m)} m_{[I} \ell_{J]}-2 U_{a}^{(l, m)} \bar{m}_{[I} l_{J]}+R_{I J}
$$

where, the one form $R_{I J}$ is such that $R_{I J} n^{J} \triangleq 0$. For the null vector $m_{I}$, we get:

$$
A_{\leftarrow}^{a} I J \triangleq-2 U_{a}^{(\ell, m)} \bar{m}_{[I} l_{J]}+2 V_{a}^{(m)} m_{[I} \bar{m}_{J]}+S_{I J}
$$

where, the one form $S_{I J}$ is such that $S_{I J} m^{J} \triangleq 0$. A similar construction for the null vector $\bar{m}_{I}$ implies

$$
A_{\leftarrow} I J \triangleq-2 \bar{U}_{a}^{(\ell, m)} \bar{m}_{[I} l_{J]}+2 V_{a}^{(m)} m_{[I} \bar{m}_{J]}+\bar{S}_{I J}
$$

such that $\bar{S}_{I J} \bar{m}^{J} \triangleq 0$

The connections that we have obtained above complement each other. Combining all these expressions, we get the complete expression for the connection $A_{\leftarrow} I J$. These gives the connection to be:

$$
A_{I J}=-2 \omega^{(\ell)} \ell_{[I} n_{J]}+2 U^{(l, m)} \ell_{[I} \bar{m}_{J]}+2 \bar{U}^{(l, m)} \ell_{[I} m_{J]}+2 V^{(m)} m_{[I} \bar{m}_{J]}
$$

We define the following connection for ease of computation ${ }^{2}$

$$
A_{I J}^{(H)}:=\frac{1}{2}\left(A_{I J}-\frac{\gamma}{2} \epsilon_{I J}{ }^{K L} A_{K L}\right)
$$

\footnotetext{
2 This choice of the connection $A_{I J}^{(H)}$ with a factor $\frac{1}{2}$ in front is made to make our results conform to the standard results [12]. However, this factor can be arbitrarily chosen and the quantum implementation of the boundary conditions including state counting goes through unchanged entirely.
} 
This leads to the following form of the connection:

$$
\begin{aligned}
A_{a I J}^{(H)} & \triangleq \ell_{[I} n_{J]}\left(-\omega_{a}^{(\ell)}+i \gamma V_{a}^{(m)}\right)+m_{[I} \bar{m}_{J]}\left(V_{a}^{(m)}-i \gamma \omega_{a}^{(\ell)}\right) \\
& +\ell_{[I} \bar{m}_{J]}\left(U_{a}^{(l, m)}+i \gamma U_{a}^{(l, m)}\right)+\ell_{[I} m_{J]}\left(\bar{U}_{a}^{(l, m)}-i \gamma \bar{U}_{a}^{(l, m)}\right)
\end{aligned}
$$

Variation of the Action

The next step is to check the variational principle. The lagrangian that we are interested in is of the form:

$$
-16 \pi G \gamma L=\gamma \Sigma_{I J} \wedge F^{I J}-e_{I} \wedge e_{J} \wedge F^{I J}-\gamma d\left(\Sigma_{I J} \wedge A^{I J}\right)+d\left(e_{I} \wedge e_{J} \wedge A^{I J}\right) .
$$

where we have added the two boundary term just for convenience. These terms will not contribute to the equation of motion. The variation of the action on-shell will give two terms on the boundary $\Delta$. They are:

$$
\delta S(e, A)=\frac{-1}{8 \pi G \gamma} \int_{\Delta}\left(i V^{(m)}+\gamma \omega^{(\ell)}\right) \wedge \delta^{2} \epsilon
$$

We can argue that the term is zero and hence the action principle is well defined. The argument goes as follows. First of all, the field configurations over which the variations are taken are such that they satisfy the standard boundary conditions at infinity and the WIH boundary conditions at $\Delta$. The weak isolation condition implies that $£_{\ell} \omega^{(\ell)} \triangleq 0$ though there is no such condition on $V^{(m)}$. However interestingly, $d \omega^{(\ell)}$ and $d V^{(m)}$ are proportional to ${ }^{2} \boldsymbol{\epsilon}$ and hence inner product with $\ell^{a}$ of these quantities are zero. This implies that for variations among field configurations with null normals in the equivalence class, we have $£_{\xi \ell} \omega^{(\ell)} \triangleq d\left(\xi \kappa_{(\ell)}\right)$ and $£_{\xi \ell} V^{(m)} \triangleq d(\xi(\epsilon-\bar{\epsilon}))$. This implies that on the application of $£_{\xi \ell}$, the integral goes to the initial and the final cross section of $\Delta$. However, the variation of the fields for example $\delta^{2} \epsilon$ is zero at the initial and final hypersurface by the standard rules of variational principle. Thus the integral is lie dragged by any null normal in the equivalence class. In other words, the integral in zero at the initial and the final hypersurface and is lie dragged on $\Delta$. Thus, the entire integral is zero and the action principle is well defined.

\section{B. The Symplectic Structure}

The phase space for the system can be constructed. We recall that the variation of the Lagrangian produces the three form $\Theta(\delta)$, such that $\delta L=: d \Theta(\delta)$. In the present case, we have:

$$
16 \pi G \gamma \Theta(\delta)=\gamma \delta \Sigma_{I J} \wedge A^{I J}-\delta\left(e_{I} \wedge e_{J}\right) \wedge A^{I J}=-2 \delta\left(e^{I} \wedge e^{J}\right) \wedge A_{I J}^{(H)}
$$

The construction of the symplectic current from here is standard. The current is $J\left(\delta_{1}, \delta_{2}\right):=\delta_{1} \Theta\left(\delta_{2}\right)-\delta_{2} \Theta\left(\delta_{1}\right)$. The current is closed on- shell i.e. $d J=0$. The resulting Symplectic Current is :

$$
J\left(\delta_{1}, \delta_{2}\right):=\frac{1}{8 \pi G \gamma}\left\{\delta_{[1}\left(e_{1} \wedge e_{2}\right)\right\} \wedge\left\{\delta_{2]}\left(A_{I J}-\frac{\gamma}{2} \epsilon_{I J}{ }^{K L} A_{K L}\right)\right\}
$$

Integrating the symplectic current over $\mathcal{M}$, we get the contribution of the symplectic current from the boundaries of the spacetime region under consideration:

$$
\int_{M_{+} \cup M_{-} \cup \Delta \cup i^{0}} J\left(\delta_{1}, \delta_{2}\right)=0
$$

The boundary conditions at infinity ensure that the integral of the symplectic current at $i^{0}$ vanishes. However, to construct the symplectic structure, we must be careful that no data flows out of the phase space because of our choice of foliation. To ensure this, we must check that the symplectic structure is independent of the choice of foliation. For that, we introduce potentials:

$$
\begin{aligned}
& \text { 1. } £_{(\xi \ell)} \psi_{(\xi \ell)} \triangleq \xi \ell^{a} \omega_{a}^{(\xi \ell)} \triangleq \kappa_{(\xi \ell)} \\
& \text { 2. } £_{(\xi \ell)} \mu_{(m)} \triangleq i \xi \ell^{a} V_{a}^{(m)} \triangleq i \xi(\epsilon-\bar{\epsilon})
\end{aligned}
$$


along with the boundary condition that they are zero at one of the cross- sections of $\Delta$ so as to fix the additive ambiguities. We choose $\psi_{(\xi \ell)}=0$ and $\mu_{(m)}=0$ at $S_{-}$.

The basic idea now is to write the symplectic current $J\left(\delta_{1}, \delta_{2}\right)$ on $\Delta$ in terms of these potentials and see that $J\left(\delta_{1}, \delta_{2}\right) \triangleq d j\left(\delta_{1}, \delta_{2}\right)$. With this simplification, the integrals of $J\left(\delta_{1}, \delta_{2}\right)$ on $\Delta$ will be taken to the boundaries $S_{ \pm}$of $\Delta$. To see this, first note that the expression of symplectic current $J\left(\delta_{1}, \delta_{2}\right)$ on $\Delta$ is given by:

$$
\left.J\left(\delta_{1}, \delta_{2}\right)\right|_{\Delta} \triangleq \frac{-1}{8 \pi G \gamma}\left[\delta_{1}^{2} \epsilon \wedge \delta_{2}\left(i V+\gamma \omega^{(\ell)}\right)-\delta_{2}^{2} \epsilon \wedge \delta_{1}\left(i V+\gamma \omega^{(\ell)}\right)\right]
$$

The potential for $\kappa_{(\ell)}$ is $\psi_{(\ell)}$. It can be seen from the definition that $\psi_{(\ell)}$ is a function of $v$ only. The potential for $i(\epsilon-\bar{\epsilon})$ is $\mu_{(m)}$ and it can be seen again that the wedge product of the variation of $\epsilon$ and variation of $d \mu_{(\ell)}+£_{\ell} \mu_{(m)} n$ vanish. These two results imply that

$$
\left.J\left(\delta_{1}, \delta_{2}\right)\right)\left.\right|_{\Delta} \triangleq d\left[\frac{-1}{8 \pi G \gamma}\left(\delta_{1}^{2} \epsilon \delta_{2}\left(\mu_{(m)}+\gamma \psi_{(\ell)}\right)-(1 \leftrightarrow 2)\right)\right]
$$

We take a particular orientation of the spacetime foliation into account. That gives us the result that the symplectic current is independent of the foliation.

$$
\left(\int_{M_{+}}-\int_{M_{-}}\right) J\left(\delta_{1}, \delta_{2}\right) \triangleq \frac{1}{8 \pi G \gamma}\left(\int_{S_{-}}-\int_{S_{+}}\right)\left\{\delta_{1}^{2} \epsilon \delta_{2}\left(\mu_{(m)}+\gamma \psi_{(\ell)}\right)-(1 \leftrightarrow 2)\right\}
$$

The construction of symplectic current is independent of our choice foliation and hence all the phase space information can be obtained from this symplectic current by staying on any arbitrary foliation. We choose a particular Cauchy surface $M$ which intersects $\Delta$ in the sphere $S_{\Delta}$. The symplectic structure is then given by:

$$
\begin{aligned}
\Omega\left(\delta_{1}, \delta_{2}\right) & :=\frac{1}{8 \pi G \gamma} \int_{M}\left[\delta_{1}\left(e^{I} \wedge e^{J}\right) \wedge \delta_{2} A_{I J}^{(H)}-\delta_{2}\left(e^{I} \wedge e^{J}\right) \wedge \delta_{1} A_{I J}^{(H)}\right] \\
& +\frac{1}{8 \pi G \gamma} \int_{S_{\Delta}}\left[\delta_{1}{ }^{2} \epsilon \delta_{2}\left(\mu_{(m)}+\gamma \psi_{(\ell)}\right)-\delta_{2}{ }^{2} \epsilon \delta_{1}\left(\mu_{(m)}+\gamma \psi_{(\ell)}\right)\right]
\end{aligned}
$$

\section{THE FIRST LAW}

The first law requires defining an energy. Since the WIH is a local definition of a horizon, the first law should involve only locally defined quantities. To be more precise, the first law is expected to relate variations of local quantities that are defined only at the horizon without any reference to the rest of the spacetime. We already have the surface gravity $\kappa_{(\xi \ell)}$ defined only locally at the horizon and the other quantity that we require now is a locally defined energy (for horizons carrying other charges, such as angular momentum, electric potential etc., we must also provide local definitions for them). To proceed, it should be noted that in spacetime, energy is associated with a timelike Killing vector field. Given any vector field $W$ in spacetime, it naturally induces a vector field $\delta_{W}$ in the phase space. The phase space vector field $\delta_{W}$ is the generator of time translation in the phase space. If time translation is a canonical transformation in the phase space then $\delta_{W}$ defines a Hamiltonian function $H_{W}$ for us.

So to find out the Hamiltonian function associated with energy, we must look for phase space transformations that keep the symplectic structure invariant, in other words the canonical transformations. The vector fields tangent to these canonical flows are the Hamiltonian vector fields. To check wheather a vector field $\delta_{t}$ in the phase space is Hamiltonian, one constructs a one-form $X_{t}$ where $X_{t}(\delta):=\Omega\left(\delta, \delta_{t}\right)$, where $\delta_{t}$ is the lie flow $£_{t}$ generated by the spacetime vector field $t^{a}$ when tensor fields are varied. The necessary and sufficient condition for the vector field $\delta_{t}$ to be a globally Hamiltonian vector field is that the one-form $X_{t}$ is to be exact, $X_{t}=\mathbf{d} H_{t}$ where $\mathbf{d}$ is the exterior derivative in phase space and $H_{t}$ is the corresponding Hamiltonian function. In other words, the vector field $\delta_{t}$ is globally Hamiltonian if and only if $X_{t}(\delta)=\delta H_{t}$ for any vector field $\delta$ in the phase space. Because of the presence of the boundary, the WIH, The vector fields $t^{a}$ are also restricted by the condition that it should be tangential on $\Delta$. Now being a null surface, the WIH has only three tangential directions, one null and the two other spacelike. The closest analog of 'time' translation on WIH is therefore translation along the null direction. It is generated by the vector field $\left[\xi \ell^{a}\right]$ (one can take any but fixed $\xi$ belonging to the equivalence class). For global solutions this null normal vector field becomes timelike outside the horizon and is expected to match with the asymptotic time-translation for asymptotically flat spacetimes. So in this sense, the local energy on the horizon is the local snapshot of ADM or Bondi energy defined at spacelike/null asymptotic infinity.

The relevant question is: Is the flow generated by the phase space vector field $\delta_{\xi \ell}$ Hamiltonian? To find that, we calculate the symplectic structure for any arbitrary live vector field $\delta_{\xi \ell}$. It is useful to recall that the action of the phase 
space vector field $\delta_{\xi \ell}$ on tensor fields is the lie flow $£_{\xi \ell}$ generated by the vector field $\xi \ell^{a}$. For the above symplectic structure, $X_{(\xi \ell)}(\delta)$ gets contribution from both the bulk and the surface symplectic structure. The bulk term, thanks to the equation of motion satisfied by the fields and their variations, contributes only through the boundaries of the Cauchy surface $M$, which are the $2-$ spheres $S_{\Delta}$ and $S_{\infty}$ respectively:

$$
\left.X_{\xi \ell}(\delta)\right|_{M}=\frac{-1}{8 \pi G} \xi \kappa_{(\ell)} \delta \mathcal{A}_{\Delta}-\frac{i}{8 \pi G \gamma} \int_{S_{\Delta}} \xi(\epsilon-\bar{\epsilon}) \delta^{2} \epsilon+\delta E_{(\xi \ell)}
$$

where, $\mathcal{A}_{\Delta}=\int_{S_{\Delta}} \epsilon$ is the area of $S_{\Delta}$ and $E_{(\xi \ell)}$ is the ADM energy arising out of the integral at $S_{\infty}$, assuming that the asymptotic time translation matches with the vector field $\xi \ell^{a}$ at infinity.

The $X_{(\xi \ell)}(\delta)$ also gets contribution from the surface symplectic structure. For $t=\xi \ell^{a}$, we must be careful with the evaluations of the action of $\delta_{(\xi \ell)}$ on the potentials $\mu_{(m)}$ and $\psi_{(\ell)}$. The action of $\delta_{(\xi \ell)}$ cannot be interpreted as $£_{(\xi \ell)}$ when acting on potentials. To determine the action, we proceed as follows. For the case of $\psi_{(\ell)}$, it is clear that since variation of $\psi_{(\xi \ell)}$ is completely determined by $\kappa_{(\xi \ell)}, \delta_{(\xi \ell)} \psi_{(\xi \ell)}=0$. However, $\psi_{(\xi \ell)}=\psi_{(\ell)}+\ln \xi$ implies that $\delta_{(\xi \ell)} \psi_{(\ell)}=-£_{\ell} \xi$. For the other potential, observe that $\delta_{(\xi \ell)} \mu_{(m)}-i(\epsilon-\bar{\epsilon})$ satisfies the differential equation $£_{\xi \ell}\left(\delta_{(\xi \ell)} \mu_{(m)}-i(\epsilon-\bar{\epsilon})\right)=0$ with the boundary condition that $\mu_{(m)}=0$ at the point $v=0$. This implies that because $(\epsilon-\bar{\epsilon})=0$ at $v=0$, the action is $\delta_{(\xi \ell)} \mu_{(m)}=i(\epsilon-\bar{\epsilon})$. The considerations above leads to:

$$
\begin{aligned}
\left.X_{\xi \ell}(\delta)\right|_{S_{\Delta}} & =\frac{1}{8 \pi G \gamma} \int_{S_{\Delta}}\left[\delta^{2} \epsilon \delta_{\xi \ell}\left(\mu_{(m)}+\gamma \psi_{(\ell)}\right)-£_{\xi \ell}{ }^{2} \epsilon \delta\left(\mu_{(m)}+\gamma \psi_{(\ell)}\right)\right] \\
& =-\frac{1}{8 \pi G} £_{\ell} \xi \delta \mathcal{A}_{\Delta}+\frac{i}{8 \pi G \gamma} \int_{S_{\Delta}} \xi(\epsilon-\bar{\epsilon}) \delta^{2} \boldsymbol{\epsilon}
\end{aligned}
$$

Combining the two equations (4.1) and (4.2), we get:

$$
X_{\xi \ell}(\delta) \triangleq-\frac{1}{8 \pi G} \kappa_{(\xi \ell)} \delta \mathcal{A}_{\Delta}+\delta E_{(\xi \ell)}
$$

This is a fundamental result of the generalization to the most general class of null normals $\left[\xi \ell^{a}\right]$. In the constant class of null normals, there is no contribution from the surface symplectic structure. In the generalized class of null normals, the precise contribution from the bulk (4.1) and the boundary (4.2) leads to the physically meaningful variation.

The condition that $\delta_{\xi \ell}$ is a Hamiltonian implies that the surface gravity $\kappa_{(\xi \ell)}$ is a function of area $\mathcal{A}_{\Delta}$ only. To see that note that to check that $\delta_{\xi \ell}$ is a Hamiltonian, we must check that $\mathbf{d} X_{\xi \ell}=0$. In other words, this implies $\mathbf{d} X_{\xi \ell}\left(\delta_{1}, \delta_{2}\right)=0$. A simple calculation gives:

$$
\begin{aligned}
\mathbf{d} X_{\xi \ell}\left(\delta_{1}, \delta_{2}\right) & =\delta_{1}\left(\kappa_{(\xi \ell)} \delta_{2} \mathcal{A}_{\Delta}\right)-\delta_{2}\left(\kappa_{(\xi \ell)} \delta_{1} \mathcal{A}_{\Delta}\right) \\
& =\delta_{1} \kappa_{(\xi \ell)} \delta_{2} \mathcal{A}_{\Delta}-\delta_{2} \kappa_{(\xi \ell)} \delta_{1} \mathcal{A}_{\Delta}
\end{aligned}
$$

This can be written in a more suggestive form as $\mathbf{d} \kappa_{(\xi \ell)} \wedge \mathbf{d} \mathcal{A}_{\Delta}\left(\delta_{1}, \delta_{2}\right)=0$. This implies that since $\delta_{1}$ and $\delta_{2}$ are arbitrary, the wedge product is zero by itself i.e. we get:

$$
\mathbf{d} \kappa_{(\xi \ell)} \wedge \mathbf{d} \mathcal{A}_{\Delta}=0
$$

which implies that the surface gravity $\kappa_{(\xi \ell)}$ is a function of area $\mathcal{A}_{\Delta}$ only. The exact functional form however remains undetermined. This also implies that there exists a locally defined function $E_{\Delta}$. Defining the total Hamiltonian $X_{\xi \ell}(\delta)=\delta H_{\xi \ell}=: E_{(\xi \ell)}-E_{\Delta}$, we get the first law of weak isolated horizons:

$$
\delta E_{\Delta} \triangleq \frac{1}{8 \pi G} \kappa_{(\xi \ell)} \delta \mathcal{A}_{\Delta}
$$

This is consistent since the previous condition imples the existence of a locally defined energy such that $\mathbf{d} \kappa_{(\xi \ell)} \wedge$ $\mathbf{d} \mathcal{A}_{\Delta}\left(\delta_{1}, \delta_{2}\right)=0$. With the present first law, we see that this relationship holds.

\section{CHERN-SIMONS THEORY FROM SYMPLECTIC STRUCTURE}

Once the four laws of black hole mechanics are established one wonders whether their resemblances with the four laws of thermodynamics are pure coincidences? In a brilliant paper, Bekenstein argued that this is not a mere 
resemblance but black holes indeed may have entropy proportional to the classical area of the horizon. Shortly after, by analyzing quantum fields in a collapsing spacetime Hawking showed that a black hole has a temperature of (in units of $\left.\hbar=k_{B}=1\right) \kappa / 2 \pi$, where $\kappa$ is the surface gravity at the horizon. It readily implies that under semiclassical approximation the entropy of a black hole is equal to the one-quarter of its horizon area. This interpretation of entropy as area obviously begs for its derivation from first principles, namely the Boltzmann definition of entropy arising out the microstates of the system. However, in the present case the system being a black hole spacetime, the question is what are its microstates? In other words, what are the microscopic constituents of a black hole spacetime to which such microstates are to be assigned? This is indeed a deep question and its answer lies beyond the general theory of relativity. Since the laws of the microscopic world are quantum mechanical, one naturally asserts that such microscopic constituents of spacetime obey the laws of quantum mechanics, rather than the classical laws of general relativity. This therefore, warrants for a quantum theory of spacetime or a quantum theory of gravity. Such a theory, to our complete satisfaction, is simply not available at present.

One general approach towards a statistical interpretation of black hole entropy is the loop approach which flourished under the umbrella of loop quantum gravity. In this approach, the statistical analysis of the microscopic constituents for a generic black hole was first presented in a series of papers [11-13]. The general idea was to not to find out the microscopic constituents of an entire black hole spacetime, but rather their imprints on the classical horizon of the black hole, which when treated as a boundary of the spacetime outside of a black hole, gets excited by some effective degrees of freedom that arise due to a delicate but well-defined interaction between the boundary and the bulk of the spacetime. One assumes that these effective degrees of freedom capture the bare minimal features of a black hole spacetime, thereby it is only natural that such effective states are localized only at the horizon rather than spread out all over the spacetime. The isolated horizons become relevant in this context because such surfaces are tailored to capture the essential features of a black hole spacetime. One then quantizes this effective theory induced at an isolated horizon and and count the appropriate quantum states. This turn out to be consistent with the semiclassical estimates made by Bekenstein and Hawking. Furthermore, the effective theory on the horizon can only be a theory of the topological kind, namely it must be insensitive to the metric on the horizon. This is because the horizon is a null surface and therefore cannot support a physical particle. The above papers show, through a detailed canonical phase space analysis, that the effective theory on the horizon is Chern-Simons type, more precisely a $U(1)$ Chern-Simons theory.

The main objective of the section is to find out the effective field theory on a spherically symmetric WIH, starting from the Holst action, in a completely covariant framework. It will not only reinforce the claims made in the above work but also at the same time will make the results independent of any slicing.

\section{A. Spherical Horizons}

The plan is to use these considerations to find the toplogical theory on the inner boundary called WIH. To proceed further, we consider the following case: the fields on the boundary are such that the energy mommentum tensor is of the form $-T_{a}^{b} \ell^{a}=e \ell^{b}$, where $e$ is spherically symmetric. Then, using Einstein equation, we get:

$$
\Phi_{11}+\frac{1}{8} R-\frac{1}{2} \Lambda \triangleq 4 \pi G e
$$

This condition implies that for spacetimes with cosmological constant zero, the term $\Phi_{11}+\frac{1}{8} R$ is spherically symmetric as $e$ is spherically symmetric. From the above energy conditions with the above form of $-T_{a}^{b} \ell^{a}$ it also follows simply from the Einstein equations that $\Phi_{00} \triangleq \Phi_{01} \triangleq \Phi_{10} \triangleq 0$. This implies that no flux of radiation falls through the horizon. This is expected as the spherical symmetric energy condition is a special case of the more general cases considered so far.

We need one further expression to proceed further. First, see that for spherical symmetric horizons $\pi \triangleq 0$ and $\lambda \triangleq 0$ and $\mu$ is a real and spherically symmetric function. In fact, in canonical formulation, this function measures the expansion of the null vector $n$. In general black hole horizons, the function $\mu$ is positive but might not be spherically symmetric. Indeed, for distorted black hole horizons, it is true that $\mu$ is not spherically symmetric and $\pi$ is not zero. The Riemann tensor is then calculated for the null vector $n^{a}$ by using (3.11) as follows:

$$
\begin{aligned}
R_{a b c d} n^{d}=2 \nabla_{[a} \nabla_{b]} n^{c} & \triangleq 2 \nabla_{[a} \omega_{b]} n_{c}+2 \partial_{[a} \mu \bar{m}_{b]} m_{c}+2 \mu \nabla_{[a} \bar{m}_{b]} m_{c} \\
& +2 \mu \bar{m}_{[b} \nabla_{a]} m_{c}+2 \partial_{[a} \mu m_{b]} \bar{m}_{c}+2 \mu \nabla_{[a} m_{b]} \bar{m}_{c} \\
& +2 \mu m_{[b} \nabla_{a]} \bar{m}_{c}+2 \mu \omega_{[a} \bar{m}_{b]} m_{c}+2 \mu \omega_{[a} m_{b]} \bar{m}_{c}
\end{aligned}
$$

We can use the expansion of the Riemann tensor in terms of the Weyl tensor, the Ricci tensor and the Ricci scalar. 
Transvecting with $\ell^{a} m^{b} \bar{m}^{c}$, and using the expansion of the scalar $\Phi_{11}$, we get that:

$$
\mathbf{\Psi}_{2}+\frac{1}{12} R \triangleq £_{\ell} \mu+\kappa_{(\ell)} \mu
$$

This equation then implies that since the all the terms on the right hand side are real and $R$ is real, the term $\operatorname{Im} \Psi_{2}=0$, i.e., $d \omega^{(\ell)}=0$. Also, the equation implies that the term $\left(\operatorname{Re} \boldsymbol{\Psi}_{\mathbf{2}}+\frac{1}{12} R\right)$ is again spherically symmetric as $\mu$ is spherically symmetric.

To proceed further, we want to restrict ourselves to the fixed area phase space. This needs an expression of the area two- form to be fixed on the phase space. We have already noticed that for spherical horizons, $d \omega^{(\ell)}=0$. However, the curvature of the one form $V^{(m)}$ involves the area form. The details of the calculation is given in the appendix (see appendix IX C). Using the equations (5.3) and (5.1), we see that the term $\mathcal{F}:=\left(\mathbf{\Psi}_{\mathbf{2}}{ }^{(H)}-\Phi_{11}-\frac{R}{24}\right)$ in the equation (9.27) is again spherically symmetric. This property of $\mathcal{F}$ remains to be spherically symmetric throughout $\Delta$. To see this, note that the Bianchi identity for $V^{(H)}$ in equation (9.27) implies:

$$
d \mathcal{F} \wedge{ }^{2} \epsilon \triangleq 0
$$

where, we have used that $d^{2} \boldsymbol{\epsilon} \triangleq 0$. Transvected by $\ell^{a}$, the above equation shows that the value of $\left(\mathbf{\Psi}_{\mathbf{2}}{ }^{(H)}-\Phi_{11}-\frac{R}{24}\right)$ is lie dragged by $\ell^{a}$ and hence, remains fixed over $\Delta$. We want to find a value for $\mathcal{F}$ in (9.27). Then, we first evaluate the curvature of the connection $V^{(H)}=-i m_{[I} \bar{m}_{J]} A^{(H) I J}$ by using the Gauss- Bonnet theorem. The connection $i V^{(m)}$ is precisely the connection on the sphere $S^{2}$ and then its field strength will be the curvature. Integrating both sides of (9.27), using Gauss- Bonnet theorem and remembering that $\mathcal{F}$ is independent of sphere coordinates, we get:

$$
\left(\Psi_{\mathbf{2}}{ }^{(H)}-\Phi_{11}-\frac{R}{24}\right)=-\frac{2 \pi}{\mathcal{A}^{s}}
$$

This equation (5.5) and the equation (9.27) together imply that the two- form ${ }^{2} \boldsymbol{\epsilon}$ can be written in terms of the curvature of the $U(1)$ field $V^{(H)}$ :

$$
{ }^{2} \boldsymbol{\epsilon}=-\frac{\mathcal{A}_{\Delta}^{s}}{2 \pi} d V_{s}^{(H)}
$$

The superscripts and subscripts $s$ in $\mathcal{A}_{\Delta}^{s}$ and $V_{s}^{(H)}$ denote that we are in spherically symmetric case. As we shall see, this term will give the requisite level for the $U(1)$ Chern- Simons theory. One can envisage another approach. Consider all spacetimes which provide different set of values for $\left[\mathbf{\Psi}_{\mathbf{2}}{ }^{(H)}-\Phi_{11}-\frac{R}{24}\right]$. Now, we shall consider only the average values. Then integrating the Eq.. (9.27), we get an average value of $\left[\mathbf{\Psi}_{\mathbf{2}}{ }^{(H)}-\Phi_{11}-\frac{R}{24}\right]$ to be $\frac{-2 \pi}{\mathcal{A}^{s}}$. While this is a possibility, We shall use (5.6) in future. This is the most important expression in the derivation of the $U(1)$ Chern -Simons theory. We shall use this expression for the surface contribution to symplectic current for the case when the the areas are fixed. This will imply that we will always remain in the fixed area phase space. The symplectic structure will not be able to give any first law as the variation of the area is zero on this phase space. We shall see however that the surface contribution to the symplectic structure acquires the form of a $U(1)$ Chern- Simons theory.

To prove that claim, we go back to the expression for the symplectic current. We have already seen that the symplectic current on the spacetime region bounded by the Cauchy surfaces $M_{+}, M_{-}$and $\Delta$ is given by:

$$
\left(\int_{M_{+}}-\int_{M_{-}}\right) J\left(\delta_{1}, \delta_{2}\right)=-\int_{\Delta} J\left(\delta_{1}, \delta_{2}\right)
$$

In the expression for the symplectic current on $\Delta$, i.e. $\int_{\Delta} J\left(\delta_{1}, \delta_{2}\right)$, the potentials $\psi_{(\ell)}$ and $\mu_{(m)}$ come into play(see $(3.35))$. The potential $\psi_{(\ell)}$ is a function of $v$ only while $\mu_{(m)}$ is still a function of $(\theta, \phi)$. Then on the fixed area phase space, the contribution to the symplectic current comes only from the terms involving the potential $\mu_{(m)}$. The contribution to $\Delta$ for the spherical horizons can be calculated for fixed area horizon. A simple calculation gives:

$$
\int_{\Delta} J\left(\delta_{1}, \delta_{2}\right)=\frac{1}{8 \pi G \gamma}\left(\int_{S_{-}}-\int_{S_{+}}\right)\left\{\delta_{1} \mu_{(m)} \delta_{2}^{2} \epsilon-(1 \leftrightarrow 2)\right\}
$$

The one- forms $m$ have a gauge freedom. This is given by $m(v, \theta, \phi)=e^{-i \mu_{(m)}(v, \theta, \phi)} m(0, \theta, \phi)$. From now on, we shall only indicate the $v$ dependence of $\mu_{(m)}$. This gives that $V_{(m)} \rightarrow V^{(m) g}=V^{(m)}-i d \mu_{(m)}$ (see $(3.20)$ ). The connection $V^{(H)}$ then transforms as

$$
V^{(H) g}=V^{(H)}+\frac{1}{2} d \mu_{(m)}(v)
$$


To proceed further, we use the expression (5.6) in the symplectic current and integrate by parts and again use (5.9). This gives the following expression for the current

$$
\begin{aligned}
\int_{\Delta} J\left(\delta_{1}, \delta_{2}\right) & =\frac{2}{8 \pi G \gamma} \frac{\mathcal{A}_{\Delta}^{s}}{\pi}\left(\int_{S_{-}}-\int_{S_{+}}\right)\left\{\delta_{1} V^{(H) g} \wedge \delta_{2} V^{(H) g}-(1 \leftrightarrow 2)\right\} \\
& -\frac{1}{8 \pi G \gamma} \frac{\mathcal{A}_{\Delta}^{s}}{\pi}\left(\int_{S_{-}}-\int_{S_{+}}\right)\left\{\delta_{1} V^{(H) g} \wedge \delta_{2} V^{(H)}-(1 \leftrightarrow 2)\right\}
\end{aligned}
$$

The further evaluation of the symplectic current is based on the idea that the expression for the connection $V^{(H)}$ is such that it cannot be subject to any variation as we move along the direction of $v$. This is because, this connection has $V^{(H)}$ has only the dependence on $(\theta, \phi)$. However, the information of the $v$ dependence is carried by the field $\mu_{(m)}$. This implies that $V^{(H) g}$ has all the information of the $v$ dependence. In short, the $v$ dependence of $V^{(H)} g$ has been transferred to $\mu_{(m)}$ leaving $V^{(H)}$ only with the angular dependence. Then we can reduce the expression for the symplectic current:

$$
\int_{\Delta} J\left(\delta_{1}, \delta_{2}\right)=\frac{1}{8 \pi G \gamma} \frac{\mathcal{A}_{\Delta}^{s}}{\pi}\left(\int_{S_{-}}-\int_{S_{+}}\right)\left\{\delta_{1} V^{(H) g} \wedge \delta_{2} V^{(H) g}\right\}
$$

In other words, we get, that the symplectic structure of the topological theory on WIH is precisely the symplectic structure of Chern- Simons theory. We will refer the theory on $\Delta$ to be a $U(1)$ Chern- Simons theory. The full symplectic structure for the spherically symmetric phase space of black hole spacetimes is:

$$
\Omega\left(\delta_{1}, \delta_{2}\right)=\frac{1}{16 \pi G \gamma} \int_{M}\left[\delta_{1}\left(e^{I} \wedge e^{J}\right) \wedge \delta_{2} A_{I J}^{H}-\delta_{2}\left(e^{I} \wedge e^{J}\right) \wedge \delta_{1} A_{I J}^{H}\right]-\frac{1}{8 \pi G \gamma} \frac{\mathcal{A}_{\Delta}^{s}}{\pi} \int_{S}\left\{\delta_{1} V^{(H) g} \wedge \delta_{2} V^{(H) g}\right\}
$$

The level of the Chern- Simons theory is $-\frac{1}{8 \pi G \gamma} \frac{\mathcal{A}_{\Delta}^{s}}{\pi}$. It is known that the level of the $U(1)$ Chern- Simons theory is an integer. So, we shall take $\frac{1}{8 \pi G \gamma} \frac{\mathcal{A}_{\Delta}^{s}}{\pi}$ to be a positive integer. The result is highly non trivial considered in the backdrop of the WIH formlation. What we have shown is that for all spherical horizons, extremal or non- extremal, the topological theory is still the Chern- Simons theory. The Chern- Simons gauge field does not see the $\xi$ scaling of the null normal $\ell^{a}$, which controls the value of surface gravity for the horizon. This simply implies that whatever be the null normal or whatever be the value of the surface gravity, the effective symplectic structure on the horizon is still the Chern- Simons theory.

\section{MINIMALLY COUPLED MAXWELL FIELDS ON WIH}

A horizon should also be able to hold matter fields on it. In this section, we will give a general treatment of matter fields on WIH and find out the constraints that might be placed on the matter fields. We will take the example of electromagnetic field to analyse the situation. There will be some degree of simplification for the use of Maxwell fields rather than any arbitrary matter field, but the treatment will be general in the sense that the main results will be same for all other matter fields minimally coupled to gravity. The reason we are interested in the Einstein-Maxwell system is because our main objective is analysing the nonextremal and extremal horizons through a unified formulation and the Einstein-Maxwell system provides the finest set of examples of these types of horizons. It is thus imperative to check that the WIH boundary conditions have sufficient structure to enable the existence of electromagnetic zeroth law and a first law.

This subsections are arranged in the following way. In the first, we will recall the boundary conditions of WIH which will put restrictions on the matter fields (Maxwell field), study its consequences and introduce conserved charges defined on the cross-section of the WIH. We will in the process show the main result that electromagnetic field can flow only along the horizon and none can cross it. This is a general result and can be shown to be true for all matter fields on $\Delta$. In the next subsection, we will prove that zeroth law and the go on to prove the first law for the Einstein-Maxwell system in the third subsection.

\section{A. Constraint on fields from boundary conditions}

Let us begin by recalling the boundary conditions of WIH which are of importance here. The only way that WIH boundary conditions can restrict matter is through conditions on the stress-energy tensor $T_{a b}$. Thus, constraints on 
matter fields will essentially come from the NEH boundary condition since further restriction of NEH to WIH only restricts the class of functions that can multiply the null normal $\ell^{a}$, so that the zeroth law is obeyed, and hence cannot put further restrictions on matter. Now, the basic result that was obtained by the use of NEH boundary conditions and Raychaudhuri equation is

$$
R_{a b} \ell^{a} \ell^{b} \triangleq 0
$$

It was also argued that this result holds for any null normal in the equivalence class $\left[\xi \ell^{a}\right]$. We had already pointed out that this implies, $R_{a} b \ell^{b} \triangleq 0$, Eq.. for any null normal in $\left[\xi \ell^{a}\right]$. The consequences of these are

$$
\begin{aligned}
& \Phi_{00}=\frac{1}{2} R_{a b} \ell^{a} \ell^{b} \triangleq 0 \\
& \Phi_{01}=\frac{1}{2} R_{a b} \ell^{a} m^{b} \triangleq 0 \\
& \Phi_{10}=\frac{1}{2} R_{a b} \ell^{a} \bar{m}^{b} \triangleq 0
\end{aligned}
$$

These are the basic results and are true for all the matter fields on the horizon and any null-normal in $\left[\xi \ell^{a}\right]$. However, it is useful to study these on a case by case basis for each of the matter fields since, as will be shown below, the form of the Maxwell energy -momentum tensor introduces further simplifications. To check those, let us notice that the Einstein field equation and Eq.. (6.1) implies that:

$$
T_{a b} \ell^{a} \ell^{b} \triangleq 0
$$

for any $\ell^{a}$ in $\left[\xi \ell^{a}\right]$. An immediate consequence of this and Eq.. is that

$$
T^{a}{ }_{b} \ell^{b} \triangleq-e \ell^{a},
$$

for some non negative function $e$ on $\Delta$ and any $\ell^{a}$ in $\left[\xi \ell^{a}\right]$. As we shall explicitly show and also is clear, this result physically implies that there is no flux of radiation crossing the horizon, implying isolation.

Let us now concentrate on the case of electromagnetic field. We will denote the electromagnetic counterparts by bold letters. The main condition on the field is thus

$$
\mathbf{T}_{a b} \ell^{a} \ell^{b} \triangleq 0
$$

for any $\ell^{a}$ in $\left[\xi \ell^{a}\right]$. The stress-energy tensor for electromagnetic fields is given in terms of the field strength $\mathbf{F}=d \mathbf{A}$ as

$$
\mathbf{T}_{a b}=\frac{1}{4 \pi}\left[\mathbf{F}_{a c} \mathbf{F}_{b}^{c}-\frac{1}{4} g_{a b} \mathbf{F}_{c d} \mathbf{F}^{c d}\right] .
$$

Let us now argue what to expect. Contracting Eq..(6.6) with $\ell^{a}$ on both the free indices, Eq. (6.4) implies that the vector $\ell^{a} \mathbf{F}_{a c}$ is null. Moreover, since $\mathbf{F}$ is antisymmetric, the vector is also normal to $\ell^{a}$. Thus, we can conclude that $\ell^{a} \mathbf{F}_{a c}$ is proportional to $\ell_{a}$ and hence $\left.\ell\right\lrcorner \mathbf{F} \triangleq 0$. This result will obviously be true for any $\ell^{a}$ in $\left[\xi \ell^{a}\right]$. To check this result explicitly, we contract the expression of $\mathbf{T}_{a b}$ with $\ell^{a} \ell^{b}$ for a fixed $\ell^{a}$ in $\left[\xi \ell^{a}\right]$ and check consequences for $\mathbf{F}$. With Eq.. (6.5) in mind, the first term in Eq..(6.6) can be written as

$$
\begin{aligned}
\mathbf{F}_{a c} \mathbf{F}_{d b} \ell^{a} \ell^{b} g^{c d} & =\mathbf{F}_{a c} \mathbf{F}_{d b} \ell^{a} \ell^{b}\left(m^{c} \bar{m}^{d}-\bar{m}^{c} m^{d}\right) \\
& =2\left(\mathbf{F}_{a c} \ell^{a} m^{c}\right)\left(\mathbf{F}_{a c} \ell^{a} \bar{m}^{c}\right)
\end{aligned}
$$

We have used the fact that the metric at the horizon can be expressed in terms of a null-tetrad as $g_{a b}=-2 \ell_{(a} n_{b)}+$ $2 m_{(a} \bar{m}_{b)}$ in the first step and the anti-symmetry of $\mathbf{F}$ in the second. Similarly, it can be checked that the second term in the Eq..(6.6) vanishes resulting in

$$
0 \triangleq \mathbf{T}_{a b} \ell^{a} \ell^{b} \triangleq\left|\ell^{a} m^{b} \mathbf{F}_{a b}\right|^{2},
$$

An immediate consequence of eqn. (6.8) is that $\mathbf{F}_{a b} \ell^{a}=a \ell_{b}+b n_{b}$, where $a$ and $b$ are some arbitrary functions. Contraction with $\ell^{b}$ and use of antisymmetry property of $\mathbf{F}$ implies that $b=0$ and hence, we get

$$
\stackrel{\ell^{a} \mathbf{F}_{a b}}{\longleftarrow} \triangleq 0
$$


for any $\ell^{a}$ in $\left[\xi \ell^{a}\right]$. In order to obtain a similar expression for ${ }^{*} \mathbf{F}$ recall that the stress energy tensor can be written as

$$
\mathbf{T}_{a b}=-\frac{1}{4 \pi}\left[{ }^{*} \mathbf{F}_{a c}{ }^{*} \mathbf{F}_{b}{ }^{c}-\frac{1}{4} g_{a b}{ }^{*} \mathbf{F}_{c d}{ }^{*} \mathbf{F}^{c d}\right]
$$

Using arguments which led to eqn. (6.9), we obtain a similar restriction on ${ }^{*} \mathbf{F}$ :

$$
\stackrel{\ell^{a *} \mathbf{F}_{a b}}{\longleftarrow} \triangleq 0
$$

It is straightforward to show that eqn. (6.9) and eqn. (6.11) puts further constraints on the electromagnetic field tensor. To observe this, note that one can write $F_{a b} \ell^{a} \triangleq a \ell_{b}$, for any $\ell^{a}$ in $\left[\xi \ell^{a}\right]$ and a similar one for the dual ${ }^{*} \mathbf{F}$. Then using the expressions (6.6) and (6.10), the following conditions can be easily checked.

$$
\begin{aligned}
\mathbf{T}_{a b} \ell^{a} m^{b} \triangleq 0 \triangleq \mathbf{T}_{a b} \ell^{a} \bar{m}^{b} \\
\mathbf{T}_{a b} m^{a} m^{b} \triangleq 0 \triangleq \mathbf{T}_{a b} \bar{m}^{a} \bar{m}^{b} .
\end{aligned}
$$

It is interesting to observe that the first two set of conditions are none other than the ones we already had obtained in eqn. (6.2) and thus are universal for any matter field on WIH. The second set of conditions however are special for the electromagnetic fields. In terms of Newman-Penrose components, these imply the following restrictions on the Ricci tensor:

$$
\begin{aligned}
& \Phi_{02}:=\frac{1}{2} R_{a b} m^{a} m^{b} \triangleq 0 \\
& \Phi_{20}:=\frac{1}{2} R_{a b} \bar{m}^{a} \bar{m}^{b} \triangleq 0
\end{aligned}
$$

Now, we can make some statements about the isolation of the WIH. Given a global timelike Killing vector field $\tau^{a}$, the Poynting vector, describing the direction of energy flow, is defined as $T^{a}{ }_{b} \tau^{b}$. On a WIH, the corresponding term can be defined as $T^{a}{ }_{b} \ell^{b}$ which by the boundary conditions is future directed and causal (null). Using eqn. (6.5) and the first set in eqn. (6.13), we can safely say that $T^{a}{ }_{b} \ell^{b}$ is proportional to $\ell^{a}$. In a local coordinate system adapted to WIH, $\ell^{a}=\left(\frac{\partial}{\partial v}\right)^{a}$. Thus the direction of the energy flow is along the horizon and nothing can cross the WIH though there is no restriction on the presence of radiation even arbitrary close to the horizon. For e.g., in a local advanced Eddington-Finkelstein coordinates $(v, r, \theta, \phi)$, it is easy to show that the components $F_{r v}, F_{r \theta}, F_{r \phi}$ may exist close to the horizon and are unrestricted in the values but have no contribution when restricted and pulled back to the horizon.

Let us now define the electric and magnetic flux density two forms directed outwards. The electric flux two form is given by $\mathbf{E}_{\Delta} \triangleq{ }^{*} \mathbf{F}$ and the magnetic one by $\mathbf{B}_{\Delta} \triangleq-\mathbf{F}$. The signatures have been taken such so as to take the orientation of $S_{\Delta}$ ( $S_{\Delta}$ is a cross-section of $\Delta$, see fig. 1) into account which is defined with respect to normal pointing into the horizon. Let us first evaluate

$$
£_{\xi \ell} \mathbf{F} \triangleq \xi \ell \cdot d \mathbf{F}+d(\xi \ell \cdot \mathbf{F}) .
$$

The first term on the right hand side vanishes due to Maxwell's equations on $\Delta$, while the second term is zero due to the previous restriction on $\mathbf{F}$, eqn. (6.9). Therefore we conclude that $\mathbf{F}$ is Lie dragged by any $\ell^{a}$ in $\left[\xi \ell^{a}\right]$. An identical argument for ${ }^{*} \mathbf{F}$ leads to the analogous conclusion. Therefore we obtain

$$
£_{\xi \ell} \underset{\leftarrow}{\mathbf{F}} \triangleq 0 \quad \text { and } \quad £_{\xi \ell} \stackrel{*}{\leftarrow} \triangleq 0 .
$$

These results imply that the $2-$ forms $\mathbf{E}_{\Delta}$ and $\mathbf{B}_{\Delta}$ are "time-independent". However, these do not restrict the forms of these fields otherwise.

We can now define the electric charge of the horizon (we assume that the magnetic charges are zero, which if present, can be analogously defined). Since the horizon is an inner boundary of spacetime, the normal to a 2 -sphere cross section of the horizon will naturally be inward pointing. Bearing this in mind, we define the electric charge of the horizon as

$$
Q_{\Delta}: \triangleq-\frac{1}{4 \pi} \oint_{S_{\Delta}}{ }^{*} \mathbf{F}
$$

For the definition to be meaningful,we should ensure that the values of $Q_{\Delta}$ should be independent of the cross section of the horizon $S_{\Delta}$. This result can be anticipated since the NEH boundary conditions imply that a Killing vector field exists on $\Delta$, one expects that the charge to be independent of cross-section. Since the $2-$ forms $\mathbf{E}_{\Delta}$ and $\mathbf{B}_{\Delta}$ are "time-independent", this guarantees that $Q_{\Delta}$ is independent of the choice of cross section $S_{\Delta}$ of the horizon. Note that this result was obtained using only the boundary conditions; equations of motion in the bulk are not needed. 


\section{B. Electromagnetic Zeroth Law}

The zeroth law for electromagnetic field states that one can define a scalar potential on the horizon that is constant throughout the horizon. Thus, to establish the zeroth law for the electromagnetic case, we need to define an electric potential $\Phi$ at the horizon. For this, the electromagnetic potential $\mathbf{A}$ is gauge fixed on $\Delta$ such that

$$
£_{(\xi \ell)} \mathbf{A}_{\mathfrak{a}} \triangleq \nabla_{\underline{a}} £_{(\xi \ell)} \chi_{(\xi \ell)} .
$$

where $\chi_{(\xi \ell)}$ is arbitrary non-zero but a fixed function of $v$ alone. Following eqn. (6.17), given such an electromagnetic potential $\mathbf{A}$ we can now define the scalar potential $\Phi_{(\xi \ell)}$ at the horizon as

$$
\Phi_{(\xi \ell)} \triangleq-\xi \ell \cdot \mathbf{A}+£_{(\xi \ell)} \chi_{(\xi \ell)}
$$

In a flat spacetime, the scalar potential is defined as the time component of the gauge potential one form $\mathbf{A}$. However, this requires a gauge fixing since $\mathbf{A}$ takes values in the gauge equivalence class of addition of an exact form, $\mathbf{A} \rightarrow \mathbf{A}+d \lambda, \lambda$ being some arbitrary function. So the scalar function also suffers from a gauge ambiguity of adding a total time derivative.

Recall that the Einstein-Maxwell case (static space-times) involves the electro-static potential $\Phi$ which one typically sets $\Phi=-\tau^{a} \mathbf{A}_{a}$ where $\tau^{a}$ is the static Killing field and the gauge is chosen such that the vector potential $\mathbf{A}$ tends to zero at infinity and satisfies $\mathcal{L}_{\tau} \mathbf{A}=0$ everywhere in space-time. Note that under the electromagnetic gauge transformation $\delta_{\lambda} \mathbf{A}_{a}=\nabla_{a} \lambda$, the eqn. (6.17) reduces to

$$
£_{(\xi \ell)} \overline{\mathbf{A}}_{\underline{\leftarrow}} \triangleq £_{(\xi \ell)} \nabla_{\underline{a}}\left[\chi_{(\xi \ell)}-\lambda\right]
$$

If we gauge fix $\chi_{(\xi \ell)}=\lambda$, the definition used in static spacetime is obtained.

Thus the definition we are proposing here is completely consistent and more general than is usually used. Then eqn. (6.18) is just the standard definition of scalar potential, $\xi \ell^{a}$ playing the role of "time" on $\Delta$ and the additional term in eqn. (6.18) is just a total time derivative. It follows immediately that

$$
d \Phi_{(\xi \ell)} \triangleq 0
$$

hence $\Phi_{(\xi \ell)}$ is constant on the horizon which essentially is the electromagnetic zeroth law. In order that this is true for the entire equivalence class $\left[\xi \ell^{a}\right]$ requires the gauge fixing functions to vary in the class in a specific way, $£_{(\xi \ell)} \chi_{(\xi \ell)}-\Phi_{(\xi \ell)}=\xi\left[£_{\ell} \chi_{(\ell)}-\Phi_{(\ell)}\right]$. This restriction is to be viewed as follows: it is always possible to choose $\chi_{(\ell)}=0$ for one $\ell^{a}$ such that $\Phi_{(\ell)}$ is a constant on $\Delta$. Then for each null vector $\xi \ell^{a}$, the above restriction fixes the gauge in eqn. (6.18) such that $\Phi_{(\xi \ell)}$ remains a constant on $\Delta$. For constant rescaling of $\ell^{a}$, it is consistent to choose $\chi_{(\ell)}=0$ for all $\ell^{a}$ (like one does for the flat spacetime) but is not true for the generalized class $\left[\xi \ell^{a}\right]$. It is a nontrivial fact that even for the generalized class $\left[\xi \ell^{a}\right]$, a constant potential such as in eqn. (6.18) exists making use of the gauge ambiguity (which always exists for scalar potentials) and the boundary conditions alone.

\section{Electromagnetic First Law}

The electromagnetic part of the Lagrangian four-form is given by $8 \pi L=-\mathbf{F} \wedge * \mathbf{F}$. The variation of this Lagrangian is carried out over all As that have the expected asymptotic fall-offs and are gauge fixed on $\Delta$ as in (6.17). The key point to note is that although a surface term is needed in the gravitational part of the action, thanks to the electromagnetic zeroth law, such a term is not needed for the electromagnetic part. Proceeding as before we find a bulk and a surface symplectic structure. To extract the surface term we introduce a potential for $\Phi_{(\xi \ell)}($ just like what we did for $\left.\kappa_{(\xi \ell)}\right): £_{(\xi \ell)} \varphi_{(\xi \ell)} \triangleq-\Phi_{(\xi \ell)}$. It also suffers from an additive ambiguity which is removed by choosing $\left.\varphi_{(\xi \ell)}\right|_{S_{-}} \triangleq 0$. Then the electromagnetic part of the symplectic structure becomes

$$
\begin{aligned}
& \Omega_{\mathrm{em}}\left(\delta_{1}, \delta_{2}\right)=-\frac{1}{4 \pi} \int_{M}\left[\delta_{1} * \mathbf{F} \wedge \delta_{2} \mathbf{A}-(1 \leftrightarrow 2)\right]+ \\
& \frac{1}{4 \pi} \oint_{S_{\Delta}}\left[\delta_{1} * \mathbf{F} \delta_{2}\left(\chi_{(\xi \ell)}+\varphi_{(\xi \ell)}\right)-(1 \leftrightarrow 2)\right] .
\end{aligned}
$$

Again, we wish to evaluate $X_{(\xi \ell)}$ from the electromagnetic part of the symplectic structure. Making use of the field equations we find that the bulk symplectic structure gets contributions only through the boundaries, which equals 
$\left(£_{(\xi \ell)} \chi_{(\xi \ell)}-\Phi_{(\xi \ell)}\right) \delta Q_{\Delta}$. To evaluate the contribution from the surface symplectic structure care should be taken not to equate $\delta_{(\xi \ell)}$ with $£_{(\xi \ell)}$ for the potential $\varphi$. It turns out that $\delta_{(\xi \ell)} \varphi_{(\xi \ell)} \triangleq 0$ everywhere and the contribution is $-\delta_{(\xi \ell)} \chi_{(\xi \ell)}$. Combining contributions from the bulk and the surface, we find

$$
X_{(\xi \ell)}^{\mathrm{em}}(\delta) \triangleq-\Phi_{(\xi \ell)} \delta Q_{\Delta}
$$

Thus, the combined first law for the gravitational and electromagnetic fields agrees with the standard first law of black hole thermodynamics.

\section{E.M. Contribution to Surface Symplectic Structure}

We have already obtained that $\underset{\mathbf{F}_{a b} \ell^{a}}{\triangleq}$. This implies that:

$$
\mathbf{F}_{a b} \triangleq \bar{\alpha}_{[b} m_{a]}+\alpha_{[b} \bar{m}_{a]}+\beta_{[b} \ell_{a]}
$$

where, $\alpha$ is a complex one form and $\beta$ is a real one form such that $\mathbf{F}_{a b}$ is real. However, it is also imperative that one needs more conditions on the one forms $\alpha$ and $\beta$ to match the degree of freedom of the $\mathbf{F}$ tensor.The conditions are also obtained from the previous condition eqn (6.23). They are:

$$
\begin{aligned}
\alpha_{a} \ell^{a} \triangleq 0 \triangleq \bar{\alpha}_{a} \ell^{a} \\
\beta_{a} \ell^{a} \triangleq 0
\end{aligned}
$$

This imples that $\alpha_{a}=a m_{a}+b \bar{m}_{a}$ and $\beta_{a}=\bar{c} m_{a}+c \bar{m}_{a}$. Then, we write the expansion of $\mathbf{F}$ in terms of the geometric forms $(\ell, n, m, \bar{m})$. Then, we obtain that:

$$
\underset{\leftarrow}{\mathbf{F}_{a b}} \triangleq \bar{a} \bar{m}_{[b} m_{a]}+a m_{[b} \bar{m}_{a]}
$$

Now, note that in NP formalism, the six components of $\mathbf{F}$ are expressed in terms of the three complex scalars $\phi_{0}, \phi_{1}$ and $\phi_{2}$. It can be observed that $\phi_{0} \triangleq 0$. The other scalar is $\phi_{1}=\frac{1}{2} \mathbf{F}_{a b}\left(m^{a} \bar{m}^{b}-\ell^{a} n^{b}\right)$. We can express the above equation eqn.(6.25) in terms of the complex scalars. This gives $(a-\bar{a})=\left(\phi_{1}-\bar{\phi}_{1}\right)$. Then, the expansion is :

$$
{\underset{\mathbf{F}}{a b}}_{\longleftarrow} \triangleq-\left(2 \operatorname{Im} \phi_{1}\right)^{2} \epsilon
$$

Similarly, it can be argued that the expansion of ${ }^{*} \mathbf{F}$ is :

$$
{ }^{*} \mathbf{F}_{a b} \triangleq\left(2 \operatorname{Re} \phi_{1}\right)^{2} \epsilon
$$

We now argue that on the phase space of fixed parameters, the symplectic structure of Maxwell fields does not contain any surface term. To prove this, we first see that the symplectic current is given by:

$$
J\left(\delta_{1}, \delta_{2}\right)=\frac{1}{4 \pi}\left(\delta_{1}{ }^{*} \mathbf{F} \wedge \delta_{2} A-\delta_{2}{ }^{*} \mathbf{F} \wedge \delta_{1} A\right)
$$

The next crucial step that one should take is to check wheather the integral of the symplectic current, integrated over $\Delta$ (as in fig. 1) is zero on the space of solutions. In general, this is not true. However, when we consider those histories where the parameters are held fixed, the symplectic current goes to zero when integrated over $\Delta$.This means that on the parameter-fixed phase space, the Maxwell theory does not contribute to the surface symplectic structure.

\section{DISCUSSIONS}

The Weak Isolated Horizon boundary conditions had been shown to be weak enough to include the extremal and non-extremal horizons in the same phase space [15]. This extension opens up the possibility of an understanding of entropy of extremal black holes in supergravity and string theory. Extremal black holes play a fundamental role in supergravity and the string theories (see [22] for discussions and other references). These solutions possess a high degree of supersymmetry as isometries and due to some non-renormalization theorems one expects the counting of degeneracies of the associated quantum state to be protected over a range of string coupling constant which can vary from small to large values. Thus, the results obtained perturbatively, at small values of the parameter, continue to 
hold for other large values. The popular choice for calculating the entropy of such black holes is the Killing horizon $(\mathrm{KH})$ framework and the classical entropy formula suggested in [4]. Inspite of its wide use, it is to our opinion highly unsatisfactory: Firstly, the derivation requires the existence of bifurcation two-spheres and it is not clear how to generalise the framework to extremal black holes which admit no bifurcation two-sphere. In other words, the phasespace of non-extremal black holes does not include extremal solutions. Secondly, the extremal solutions arising in such theories have curvature singularities at the horizon when the extremal limit is taken. This necessarily asks for higher order stringy corrections to be taken into account [23]. To find the entropy of these extremal black holes in string theory, it is argued that entropy of these solutions are to be defined only through limits from their non-extremal counterparts (see [24] for the arguments). However, since the phase-space of non-extremal Killing horizons do not contain extremal horizons, it becomes ambiguous how such limits are to be taken. The original formulation of isolated horizons (IH) [7] bypass some of these difficulties but still it is not enough. The extremal and non-extremal black holes continue to remain in different phase spaces so far as the validity of the first law is concerned and hence, extremal limit doesn't make much sense. A new framework, called weak isolated horizons (WIH), presented in [15], removes this difficulty allowing one to take limits in the same phase space. Since in WIH, the extremal horizons are in the same class of the non-extremal ones, the entropy of an extremal black hole is automatically determined when one quantizes this WIH. Expectedly, the result is proportional to the area of the horizon. This is consistent with the claim made in [24] that the entropy of an extremal black hole must be proportional to the area of the horizon, provided one uses a phase space that contains both extremal and non-extremal global solutions. A recent paper [28] revisits some of these arguments. The basic idea behind this paper was to check wheather such expectations are borne out in a completely covariant manner.

In this paper we extended the formulation of Weak Isolated Horizons to Holst's action. The main reason for taking up this exercise is to make WIHs applicable in the framework of loop quantum gravity which makes essential use of the Holst action (See [27] for other applications of Holst's action). Through our analysis we show that the essential structures of the phase space remain unaltered from the Palatini phase space once we rewrite everything in terms of the Holst connection one-form $A^{(H)}$. Interestingly, there still exists a boundary symplectic structure, although new potentials are needed here. Both the bulk and boundary symplectic structures conspire in such a way that the first law of WIH mechanics still holds. Although the results are very similar in spirit with the ones obtained from the Palatini action, the two cases differ substantially in details which have been elaborated in this paper. Another hallmark of our approach is that compared to the earlier results which used canonical phase space our approach is completely covariant. As a by-product we find that the effective theory at the horizon is a $U(1)$ Chern-Simons theory which is obtained here from a completely covariant framework (this is to our knowledge has not been derived earlier). We also argued that the presence of electromagnetic fields on WIH does not affect the boundary symplectic structure and hence does not have any affect on the topological theory on the boundary.

\section{ACKNOWLEDGMENTS}

We gratefully acknowledge discussions with Parthasarathi Majumdar and Parthasarathi Mitra in our group meetings. We especially thank the former for continuous encouragement and many stimulating remarks that helped to improve our work.

\section{APPENDICES}

\section{A. The Newman-Penrose formalism}

In this part of the appendix, we give a brief summary of the Newman- Penrose (NP) formalism ([25]). The details of the formalism, notation and other technicalities are given in $([1,26])$. Though we will follow [1] so far as the notation is concerned, the signature $(-,+,+,+)$, is different in our case (and is same as $[7,15])$ and hence one needs to recalculate some of the results.

The NP formalism relies on the fact that one is allowed to have a basis with set of 4 null vectors $(\ell, n, m, \bar{m})$. The pair $\ell$ and $n$ are real while pair $m$ and $\bar{m}$ are complex conjugates of each other. These satisfy the following orthonormality conditions:

$$
\ell \cdot n=-1 \quad m \cdot \bar{m}=1,
$$

the rest being equal to zero. The next step is to define the Newman-Penrose spin coefficients (also called the Ricci rotation coefficients in the tetrad formalism). All the information that the connection provides is encoded in the 12 
independent complex scalars. These are designated by special symbols as is given below:

$$
\begin{aligned}
& \kappa:=-m^{a} \ell^{b} \nabla_{b} \ell_{a}=-m^{a} D \ell_{a} \rho:=-m^{a} \bar{m}^{b} \nabla_{b} m_{a}=-m^{a} \bar{\delta} m_{a} \\
& \sigma:=-m^{a} m^{b} \nabla_{b} \ell_{a}=-m^{a} \delta \ell_{a} \mu:=-n^{a} m^{b} \nabla_{b} \bar{m}_{a}=-n^{a} \delta \bar{m}_{a} \\
& \lambda:=-n^{a} \bar{m}^{b} \nabla_{b} \bar{m}_{a}=-n^{a} \bar{\delta} \bar{m}_{a} \quad \tau:=-m^{a} n^{b} \nabla_{b} \ell_{a}=-m^{a} \Delta \ell_{a} \\
& \nu:=-n^{a} n^{b} \nabla_{b} \bar{m}_{a}=-n^{a} \Delta \bar{m}_{a} \quad \pi:=-n^{a} \ell^{b} \nabla_{b} \bar{m}_{a}=-n^{a} D \bar{m}_{a} \\
& \epsilon:=-\frac{1}{2}\left(n^{a} \ell^{b} \nabla_{b} \ell_{a}+m^{a} \ell^{b} \nabla_{b} \bar{m}_{a}\right)=-\frac{1}{2}\left(n^{a} D \ell_{a}+m^{a} D \bar{m}_{a}\right) \\
& \gamma:=-\frac{1}{2}\left(n^{a} n^{b} \nabla_{b} \ell_{a}+m^{a} n^{b} \nabla_{b} \bar{m}_{a}\right)=-\frac{1}{2}\left(n^{a} \Delta \ell_{a}+m^{a} \Delta \bar{m}_{a}\right) \\
& \alpha:=-\frac{1}{2}\left(n^{a} \bar{m}^{b} \nabla_{b} \ell_{a}+m^{a} \bar{m}^{b} \nabla_{b} \bar{m}_{a}\right)=-\frac{1}{2}\left(n^{a} \bar{\delta} \ell_{a}+m^{a} \bar{\delta} \bar{m}_{a}\right) \\
& \beta:=-\frac{1}{2}\left(n^{a} m^{b} \nabla_{b} \ell_{a}+m^{a} m^{b} \nabla_{b} \bar{m}_{a}\right)=-\frac{1}{2}\left(n^{a} \delta \ell_{a}+m^{a} \delta \bar{m}_{a}\right) .
\end{aligned}
$$

where, the symbols $D, \Delta, \delta, \bar{\delta}$ are the directional derivatives along the basis vectors $\ell, n, m, \bar{m}$, i.e.

$$
D=\ell^{a} \nabla_{a} \quad \Delta=n^{a} \nabla_{a} \quad \delta=m^{a} \nabla_{a} \quad \bar{\delta}=\bar{m}^{a} \nabla_{a} .
$$

In other words, the derivative operator written in terms of these symbols turn out to be:

$$
\nabla_{a}=-n_{a} D-\ell_{a} \Delta+\bar{m}_{a} \delta+m_{a} \bar{\delta} .
$$

The ten independent components of the Weyl tensor are expressed in terms of five complex scalars $\Psi_{0}, \Psi_{1}, \Psi_{2}, \Psi_{3}$ and $\Psi_{4}$. The ten components of the Ricci tensor are defined in terms of four real and three complex scalars $\Phi_{00}, \Phi_{11}$, $\Phi_{22}, \Lambda, \Phi_{10}, \Phi_{20}$ and $\Phi_{21}$. These scalars are defined as follows:

$$
\begin{aligned}
& \Psi_{0}=C_{a b c d} \ell^{a} m^{b} \ell^{c} m^{d} \quad \Phi_{01}=\frac{1}{2} R_{a b} \ell^{a} m^{b} \quad \Phi_{10}=\frac{1}{2} R_{a b} \ell^{a} \bar{m}^{b} \\
& \Psi_{1}=C_{a b c d} \ell^{a} m^{b} \ell^{c} n^{d} \quad \Phi_{02}=\frac{1}{2} R_{a b} m^{a} m^{b} \quad \Phi_{20}=\frac{1}{2} R_{a b} \bar{m}^{a} \bar{m}^{b} \\
& \Psi_{2}=C_{a b c d} \ell^{a} m^{b} \bar{m}^{c} n^{d} \quad \Phi_{21}=\frac{1}{2} R_{a b} \bar{m}^{a} n^{b} \quad \Phi_{12}=\frac{1}{2} R_{a b} m^{a} n^{b} \\
& \Psi_{3}=C_{a b c d} \ell^{a} n^{b} \bar{m}^{c} n^{d} \quad \Phi_{00}=\frac{1}{2} R_{a b} \ell^{a} \ell^{b} \quad \Phi_{11}=\frac{1}{4} R_{a b}\left(\ell^{a} n^{b}+m^{a} \bar{m}^{b}\right) \\
& \Psi_{4}=C_{a b c d} \bar{m}^{a} n^{b} \bar{m}^{c} n^{d} \quad \Phi_{22}=\frac{1}{2} R_{a b} n^{a} n^{b} \quad \Lambda=\frac{R}{24}
\end{aligned}
$$

To express the Weyl tensor in terms of the five complex scalars, we proceed as follows [1]. First, we construct a product of four quantities such that it has all the symmetries of the Weyl tensor. It should be antisymmetric in the first two indices and the last two indices and should remain unchanged under simultaneous interchange of the first two and the last two indices. We denote that by \{\} . For example,

$$
\begin{aligned}
\left\{A_{a} B_{b} C_{c} D_{d}\right\}: & =A_{a} B_{b} C_{c} D_{d}-B_{a} A_{b} C_{c} D_{d}-A_{a} B_{b} D_{c} C_{d}+B_{a} A_{b} D_{c} C_{d} \\
& +C_{a} D_{b} A_{c} B_{d}-D_{a} C_{b} A_{c} B_{d}-C_{a} D_{b} B_{c} A_{d}+D_{a} C_{b} B_{c} A_{d}
\end{aligned}
$$

There is subtlety in this construction of \{\} . In case all the terms in the braces are different from each other, just as in the above example all the terms are distinct, the \{\} is of the form as given above. In case the term is like $\left\{A_{a} B_{b} A_{c} B_{d}\right\}$, having some indistinguishable terms, only the first set of four terms as given above will suffice and it will satisfy the criteria of being antisymmetric in the first two indices and the last two indices and should remain unchanged under simultaneous interchange of the first two and the last two indices. It is then trivial to check that the components of the Weyl tensor $C_{a b c d}$ can be expanded as

$$
\begin{aligned}
C_{a b c d} & =F_{1212}\left\{\ell_{a} n_{b} \ell_{c} n_{d}\right\}+F_{3434}\left\{m_{a} \bar{m}_{b} m_{c} \bar{m}_{d}\right\}+F_{1234}\left\{l_{a} n_{b} m_{c} \bar{m}_{d}\right\} \\
& +F_{1314}\left\{\ell_{a} m_{b} \ell_{c} \bar{m}_{d}\right\}+F_{2324}\left\{n_{a} m_{b} n_{c} \bar{m}_{d}\right\} \\
& +\left[F_{1313}\left\{\ell_{a} m_{b} \ell_{c} m_{d}\right\}+F_{2323}\left\{n_{a} m_{b} n_{c} m_{d}\right\}+F_{1213}\left\{\ell_{a} n_{b} \ell_{c} m_{d}\right\}\right. \\
& +F_{1223}\left\{\ell_{a} n_{b} n_{c} m_{d}\right\}+F_{1323}\left\{\ell_{a} m_{b} n_{c} m_{d}\right\}+F_{1324}\left\{\ell_{a} m_{b} n_{c} \bar{m}_{d}\right\} \\
& \left.+F_{1334}\left\{\ell_{a} m_{b} m_{c} \bar{m}_{d}\right\}+F_{2334}\left\{n_{a} m_{b} m_{c} \bar{m}_{d}\right\}+\text { c.c. }\right]
\end{aligned}
$$


where, $F_{1234}, \cdots$ are expansion coefficients to be determined. The $c . c$ refers to the comples conjugates. Note that the terms outside the square braces are manifestly real whereas the terms inside are complex. The complex conjugates can be written down by simultaneous interchange of $m$ to $\bar{m}$ and index number 3 to 4 .

We now determine the terms in the expansion of $C_{a b c d}$ i.e. $F_{1234}, \cdots$. It is easy to check that: $F_{2424}=\Psi_{0}$, $F_{1224}=\Psi_{1}, F_{1324}=-\Psi_{2}, F_{1213}=-\Psi_{3}$ and $F_{1313}=\Psi_{4}$. The determination of other terms in the expansion of $C_{a b c d}$ requires some other relations. Firstly, cyclicity requires that:

$$
C_{1234}+C_{1342}+C_{1423}=0
$$

The notation used here is the following: we mark $l=1, n=2, m=3, \bar{m}=4$ so that whatever index is contracted gets the above assigned value, for e.g., $C_{1234}=C_{a b c d} \ell^{a} n^{b} m^{c} \bar{m}^{d}$. Now, since $C_{a b c d}$ is trace free, it implies the following results. Firstly,

$$
C_{1314}=C_{1332}=C_{2324}=C_{2441}=0
$$

and secondly, using eqn.(9.5) we get:

$$
\begin{array}{ll}
C_{1232}=C_{3234} & C_{1231}=C_{1334} \quad C_{1241}=C_{1443} \quad C_{1242}=C_{2434} \\
C_{1212}=C_{3434} & C_{1342}=\frac{1}{2}\left(C_{1212}-C_{1234}\right)
\end{array}
$$

Now, we will use these relations. First, note that $F_{1224}=-C_{2113}=-C_{1334}$. Thus, $-C_{1334}=F_{2443}=\Psi_{1}$. Secondly, note that $\Psi_{2}^{*}=C_{1432}=-F_{1423}$. Using eqn. (9.5), $C_{1234}=-\left(\Psi_{2}-\Psi_{2}^{*}\right)$. Thus, $F_{1234}=C_{2143}=-\left(\Psi_{2}-\Psi_{2}^{*}\right)$. Also, using the last two equation in eqn. (9.7), we get, $F_{1212}=C_{2121}=\left(\Psi_{2}+\Psi_{2}^{*}\right)$ and $F_{3434}=C_{4343}==\left(\Psi_{2}+\Psi_{2}^{*}\right)$. The rest, $F_{1314}=F_{2324}=F_{1323}=F_{1424}=0$, follows from the eqn. (9.6).

Putting all these values of $F_{a b c d}$ in the eqn. (9.4), we get

$$
\begin{aligned}
C_{a b c d} & =\left(\Psi_{2}+\Psi_{2}^{*}\right)\left[\left\{\ell_{a} n_{b} \ell_{c} n_{d}\right\}+\left\{m_{a} \bar{m}_{b} m_{c} \bar{m}_{d}\right\}\right]-\left(\Psi_{2}-\Psi_{2}^{*}\right)\left\{\ell_{a} n_{b} m_{c} \bar{m}_{d}\right\} \\
& +\left[\Psi_{4}\left\{\ell_{a} m_{b} \ell_{c} m_{d}\right\}+\Psi_{0}\left\{n_{a} \bar{m}_{b} n_{c} \bar{m}_{d}\right\}-\Psi_{2}\left\{\ell_{a} m_{b} n_{c} \bar{m}_{d}\right\}+\text { complex conjugates }\right] \\
& +\left[\Psi_{1}\left(\left\{\ell_{a} n_{b} n_{c} \bar{m}_{d}\right\}+\left\{n_{a} \bar{m}_{b} \bar{m}_{c} m_{d}\right\}\right)+\text { complex conjugates }\right] \\
& +\left[\Psi_{3}\left(\left\{\ell_{a} m_{b} m_{c} \bar{m}_{d}\right\}-\left\{\ell_{a} n_{b} \ell_{c} n_{d}\right\}\right)+\text { complex conjugates }\right]
\end{aligned}
$$

These has been used used in finding the expression for $d \omega^{(\ell)}$ and $d V^{(m)}$.

\section{B. Equivalence of Palatini and Holst Symplectic Structure}

Consider the case when the manifold has no boundary. Then, the symplectic structure is given by:

$$
\Omega\left(\delta_{1}, \delta_{2}\right):=\frac{1}{16 \pi G \gamma} \int_{M}\left\{\delta_{[1}\left(e_{1} \wedge e_{2}\right)\right\} \wedge\left\{\delta_{2]}\left(A_{I J}-\frac{\gamma}{2} \epsilon_{I J}{ }^{K L} A_{K L}\right)\right\}
$$

Now, the crucial point to note is the following. When viewed from the perspective of phase-space, the Holst term is a canonical transformation on the phase space. We shall show that $\gamma$ dependent term will vanish. even in presence of inner boundaries. We then must show that:

$$
\begin{aligned}
\delta_{1}\left(e^{I} \wedge e^{J}\right) \wedge \delta_{2} A_{I J} & =\delta_{2}\left(e^{I} \wedge e^{J}\right) \wedge \delta_{1} A_{I J} \\
\Rightarrow \delta_{1}\left(e^{I}\right) \wedge\left(e^{J} \wedge \delta_{2} A_{I J}\right) & =\delta_{2}\left(e^{I}\right) \wedge\left(e^{J} \wedge \delta_{1} A_{I J}\right)
\end{aligned}
$$

To prove the equality, we use the equation of motion. Firstly, from the equation of motion, we get that

$$
e^{J} \wedge \delta_{2} A_{I J}=d \delta_{2} e^{I}+A_{I J} \delta_{2} e^{J}
$$

Putting this equation eqn. (9.11) in the L.H.S. of (9.10), we get,

$$
\delta_{1}\left(e^{I}\right) \wedge\left(e^{J} \wedge \delta_{2} A_{I J}\right)=\delta_{1}\left(e^{I}\right) \wedge d\left(\delta_{2} e_{I}\right)-A_{I J} \wedge e^{I} \wedge \delta_{2} e^{J} .
$$

Also, the first term in (9.12) can be further reduced as:

$$
\delta_{1} e^{I} \wedge d \delta_{2} e_{I}=-d\left(\delta_{1} e^{I} \wedge \delta_{2} e_{I}\right)+d \delta_{1} e^{I} \wedge \delta_{2} e_{I}
$$


Thus, using the equation eqn. (9.13), the equation (9.12) becomes:

$$
\begin{aligned}
\text { L.H.S. } & =-d\left(\delta_{1} e^{I} \wedge \delta_{2} e_{I}\right)+d \delta_{1} e^{I} \wedge \delta_{2} e_{I}-A_{I J} \wedge e^{I} \wedge \delta_{2} e^{J} \\
& =-d\left(\delta_{1} e^{I} \wedge \delta_{2} e_{I}\right)+\delta_{2} e_{I} \wedge\left(d \delta_{1} e^{I}+A_{I J} \wedge \delta_{1} e^{J}\right) \\
& =-d\left(\delta_{1} e^{I} \wedge \delta_{2} e_{I}\right)+\delta_{2} e_{I} \wedge\left(e^{J} \wedge \delta_{1} A_{I J}\right) .
\end{aligned}
$$

where in the third line in the above equation, we have used the equation of motion, i.e., the $\delta_{1}$ version of eqn. (9.11). Thus, the term in the L.H.S. is equal to the term on the R.H. S. In other words, the Palatini and the Holst symplectic structure are equivalent when there is no boundary in the spacetime.

If the spacetime has an inner boundary which is the present case of interest, it is instructive to check wheather the equivalence still holds. To check that, we go back to the construction of the symplectic current $J\left(\delta_{1}, \delta_{2}\right)$. The result has been calculated before and gives

$$
J\left(\delta_{1}, \delta_{2}\right):=\frac{1}{16 \pi G \gamma}\left\{\delta_{[1}\left(e_{1} \wedge e_{2}\right)\right\} \wedge\left\{\delta_{2]}\left(A_{I J}-\frac{\gamma}{2} \epsilon_{I J}{ }^{K L} A_{K L}\right)\right\}
$$

Integrating the symplectic current over $\mathcal{M}$, taking the orientation into account, we get:

$$
\left(\int_{M_{+}}-\int_{M_{-}}\right) J\left(\delta_{1}, \delta_{2}\right)+\int_{\Delta} J\left(\delta_{1}, \delta_{2}\right)=0
$$

We have already seen that the $\gamma$-dependent term in the symplectic current gives a total derivative term. In the following steps, we will only concern ourselves with $\gamma$ - dependent term in the symplectic current since the other $\gamma$ independent term is the standard Palatini symplectic current.

The first integration of the $\gamma$ - dependent term over $M_{+}$will go to the boundaries of $M_{+}$and thence leave an integral over $S_{+}$(and at infinity which goes to zero by asymptotic boundary conditions). Similarly, the integration of the $\gamma-$ dependent term over $M_{-}$will leave an integral over $S_{-}$. The integral over $\Delta$ will give two boundary integrals, one at $S_{+}$and another at $S_{-}$. Taking the orientations of the surfaces, we get that the $\gamma$ - dependent symplectic current with $J_{\gamma}\left(\delta_{1}, \delta_{2}\right)=d \alpha\left(\delta_{1}, \delta_{2}\right)$ gives:

$$
\left(\int_{S_{+}}-\int_{S_{-}}\right) \alpha\left(\delta_{1}, \delta_{2}\right)+\left(\int_{S_{-}}-\int_{S_{+}}\right) \alpha\left(\delta_{1}, \delta_{2}\right)+\int_{M_{+} \cup M_{-} \cup \Delta} J_{P}\left(\delta_{1}, \delta_{2}\right)=0
$$

where, $J_{P}\left(\delta_{1}, \delta_{2}\right)$ is the symplectic current for the Palatini action. The above equation shows that the contributions from the $\gamma$ - dependent terms cancel. This implies that even in presence of boundaries, the Holst symplectic current is equivalent to that of Palatini. In other words, even in presence of boundaries, the canonical transformation holds good.

\section{Calculation of $d V$}

Let us consider the definition of the Riemann tensor:

$$
\left[\nabla_{a} \nabla_{b}-\nabla_{b} \nabla_{a}\right] X^{c}=-R_{a b d}^{c} X_{d}
$$

Now, consider the case when the vector $X^{a}=m^{a}$. Then, we have:

$$
\begin{aligned}
{\left[\nabla_{a} \nabla_{b}-\nabla_{b} \nabla_{a}\right] m^{c} } & \triangleq\left(\nabla_{a} U_{b}-\nabla_{b} U_{a}\right) \ell^{c}+\left(\nabla_{a} V_{b}^{(m)}-\nabla_{b} V_{a}^{(m)}\right) m^{c}+\left(\omega_{a} U_{b}-\omega_{b} U_{a}\right) \ell^{c}+\left(U_{a} V_{b}^{(m)}-U_{b} V_{a}^{(m)}\right) \ell^{c} \\
& \triangleq-R_{a b d}{ }^{c} m_{d}
\end{aligned}
$$

Multiply both sides by $\bar{m}_{c}$, we get:

$$
\left(\nabla_{a} V_{b}^{(m)}-\nabla_{b} V_{a}^{(m)}\right) \triangleq-R_{a b d}^{c} m^{d} \bar{m}_{c} \triangleq R_{a b c d} m^{d} \bar{m}^{c}
$$

Now, we use the expansion of Riemann tensor in terms of the Ricci, the Weyl tensor and the Ricci Scalar

$$
R_{a b}{ }^{c d}=C_{a b}{ }^{c d}+2 R_{[a}{ }^{[c} g_{b]}{ }^{d]}-\frac{1}{3} R g_{[a}{ }^{c} g_{b]}{ }^{d}
$$


Then, using the expansion of the the Weyl tensor in terms of the Newman-Penrose scalars (see appendix (IX A)), we get the following result:

$$
R_{a b c d} m^{d} \bar{m}^{c} \triangleq 4 \operatorname{Re} \boldsymbol{\Psi}_{\mathbf{2}} m_{[a} \bar{m}_{b]}+\frac{1}{2}\left(R_{b d} \bar{m}_{a} m^{d}-R_{b c} \bar{m}^{c} m_{a}-R_{d a} m^{d} \bar{m}_{b}+R_{c a} \bar{m}^{c} m_{b}\right)+\frac{1}{3} R m_{[a} \bar{m}_{b]}
$$

To simplify the second term in the term in the above expression of eqn. $(9.22)$, we consider the following. Let,

$$
R_{\llcorner}{ }^{d} m_{d} \triangleq A m_{b}+B \bar{m}_{b}+C n_{b} .
$$

where, $A, B$ and $C$ are to be determined. This implies that $A=R_{b d} \bar{m}^{b} m^{d}, B=R_{b d} m^{b} m^{d}$ and $C=0$ as $R_{a b} \ell^{a} m^{b} \triangleq 0$. This means,

$$
R_{b}{ }^{d} m_{d} \triangleq\left(R_{p q} \bar{m}^{p} m^{q}\right) m_{b}+\left(R_{p q} m^{p} m^{q}\right) \bar{m}_{b}
$$

Putting these expressions in the eqn. (9.22), we get

$$
d V^{(m)} \triangleq \frac{1}{i}\left[2 \operatorname{Re} \boldsymbol{\Psi}_{\mathbf{2}}-\left(R_{p q} m^{p} \bar{m}^{q}\right)+\frac{R}{6}\right]{ }^{2} \boldsymbol{\epsilon}
$$

Using the expression $\Phi_{11}=\frac{1}{4} R_{a b}\left(\ell^{a} n^{b}+m^{a} \bar{m}^{b}\right)$, it is easy to check that $\Phi_{11}=\frac{1}{8} R+\frac{1}{2} R_{a b} \ell^{a} n^{b}$. Then, defining the real connection $i V^{(m)}=: \bar{V}^{(m)}=-i m_{[I} \bar{m}_{J]} A^{I J}$, we get the following expression:

$$
d \bar{V}^{(m)} \triangleq 2\left(\operatorname{Re} \boldsymbol{\Psi}_{2}-\Phi_{11}-\frac{R}{24}\right)^{2} \boldsymbol{\epsilon}
$$

Let us now look at the expression for the connection $A_{I J}^{H}$. We shall define a new connection for this total connection by projecting $A_{I J}^{H}$ as follows. Define the following connection $V^{(H)} \triangleq-i m_{[I} \bar{m}_{J]} A^{(H) I J}$ and the curvature of this connection becomes ${ }^{3}$ :

$$
\begin{aligned}
d V^{(H)} & =\left[\operatorname{Re} \boldsymbol{\Psi}_{\mathbf{2}}+\gamma \operatorname{Im} \boldsymbol{\Psi}_{\mathbf{2}}-\Phi_{11}-\frac{R}{24}\right]{ }^{2} \boldsymbol{\epsilon} \\
& =\left[\boldsymbol{\Psi}_{\mathbf{2}}{ }^{(H)}-\Phi_{11}-\frac{R}{24}\right]{ }^{2} \boldsymbol{\epsilon}
\end{aligned}
$$

where, we have defined $\boldsymbol{\Psi}_{\mathbf{2}}{ }^{(H)}=\operatorname{Re} \boldsymbol{\Psi}_{\mathbf{2}}+\gamma \operatorname{Im} \boldsymbol{\Psi}_{\mathbf{2}}$. If we are in the vacuum $\left(\Phi_{11}\right)=0$ and the cosmological constant is zero, then we have:

$$
d V^{(H)} \triangleq \mathbf{\Psi}_{\mathbf{2}}^{(H) 2} \boldsymbol{\epsilon}
$$

\section{Going back to Canonical Phase Space}

We now use the foregoing results of the covariant phase space to get the results of the canonical phase space to make comparisons. To set the stage, we recapitulate the following conventions and results [18]:

The indices $i, j, \cdots$ take values of the subspace orthogonal to the internal fixed vector $\tau^{I}$. If the projection vector is denoted by $q_{I}^{i}$, then the induced internal metric on the subspace is given by:

$$
\eta_{i j}=q_{i}^{I} q_{j}^{J} \eta_{I J}
$$

The internal 4- dimensional antisymmetric tensor $\epsilon_{I J K L}$ naturally induces the completely antisymmetric tensor on the subspace and will be denoted by $\epsilon_{i j k}$ such that:

$$
\epsilon_{i j k}=q_{i}^{I} q_{j}^{J} q_{k}^{K} \tau^{L} \epsilon_{L I J K}
$$

\footnotetext{
${ }^{3}$ See the appendix IX D for details and other comparisons.
} 
If we define the connection $A^{(H)}{ }_{I J}$, then we can define a connection one form $\Gamma^{i}:=\frac{1}{2} q_{I}^{i} \epsilon^{I J}{ }_{K L} \tau_{J} A^{(H) K L}$ and the extrinsic curvature $K^{1}:=q_{I}^{i} A^{(H) I J} n_{J}$. Let us now define the connection $A^{(H) i}:=\Gamma^{i}-\gamma K^{i}$. It then follows that

$$
A^{(H) i}=-\frac{1}{2} \epsilon_{K L}^{i} A^{(H) K L} .
$$

To see further, let us now consider the way to define the unit time- like normal $\tau^{I}$. The obvious way is to use the null- normals $\ell^{a}$ and $n^{a}$. We define:

$$
\tau^{I}:=\frac{\left(\ell^{I}+n^{I}\right)}{\sqrt{2}} \quad r^{I}:=\frac{\left(\ell^{I}-n^{I}\right)}{\sqrt{2}}
$$

This implies that the internal vector $r^{i}$ is such that it picks out the connection intrinsic to the two sphere $S_{\Delta}$ :

$$
\begin{aligned}
A^{i} r_{i} & =-\frac{1}{2 \sqrt{2}} q_{i}^{I}\left(\ell_{I}-n_{I}\right) \epsilon_{K L}^{i} A^{(H) K L} \\
& =\frac{i}{4}\left(\ell_{K} n_{L}-\ell_{L} n_{K}\right) \epsilon^{K L}{ }_{I J} A^{(H) I J} \\
A^{i} r_{i} & =-i m_{[I} \bar{m}_{J]} A^{(H) I J}=: \tilde{V} .
\end{aligned}
$$

We can also define momentum $P_{i}^{a}=\frac{1}{16 \pi G \gamma} e_{b}^{j} e_{c}^{k} \epsilon_{i j k} \eta^{a b c}$. The 2 form dual to the momentum pulled back to $S_{\Delta}$ is given by $\sum_{a b}^{i}=\eta_{a b c} P_{j}^{a} \eta^{i j}$. Then, we have:

$$
\begin{aligned}
\Sigma_{p q}^{i} r_{i} & =\frac{1}{16 \pi G \gamma} 2 i m^{[j} \bar{m}^{k]} \epsilon_{j k}^{i} r_{i}{ }^{2} \epsilon_{p q} \\
& =\frac{1}{8 \pi G \gamma}{ }^{2} \epsilon_{p q} .
\end{aligned}
$$

This leads to the following form of the curvature on the sphere $S_{\Delta}$

$$
d V_{s}^{(H)}=-\frac{2 \pi}{\mathcal{A}_{\Delta}^{s}} 8 \pi G \gamma \Sigma^{i} r_{i}
$$

\section{E. Spherical Symmetry}

We consider the spherical symmetric metric and prove the claims that $\lambda=\pi \triangleq 0$ and $\mu$ is spherically symmetric. Consider the following most general spherically symmetric metric:

$$
d s^{2}=-f(r, t) d t^{2}+g(r, t) d r^{2}+r_{\Delta}^{2} d \Omega_{2}
$$

where, $d \Omega_{2}$ is the $2-$ sphere metric. The null normals can be calculated to as follows:

$$
\begin{aligned}
& \ell^{a} \triangleq \frac{1}{\sqrt{2}}\left(-\frac{1}{f} \frac{\partial}{\partial t}+\frac{1}{g} \frac{\partial}{\partial r}\right) \\
& n^{a} \triangleq \frac{1}{\sqrt{2}}\left(-\frac{1}{f} \frac{\partial}{\partial t}-\frac{1}{g} \frac{\partial}{\partial r}\right) \\
& m^{a} \triangleq \frac{1}{r_{\Delta} \sqrt{2}}\left(\frac{\partial}{\partial \theta}-\frac{i}{\operatorname{Sin} \theta} \frac{\partial}{\partial \phi}\right)
\end{aligned}
$$

where, the normalizations have been fixed so that $\ell \cdot n=-1$ and $m \cdot \bar{m}=1$. The covariant derivative of the null normal turns out to be:

$$
\nabla_{a} n_{b}=\partial_{a} f(d t)_{b}-f \Gamma_{a b}^{t}+\partial_{a} g(d r)_{b}-g \Gamma_{a b}^{r}
$$

Then, it follows simply that $\pi=0$ and $\lambda=0$. For $\mu$, observe that $\mu=\left(f \Gamma_{a b}^{t}+g \Gamma_{a b}^{r}\right) m^{a} \bar{m}^{b}$ will only contribute for $a, b=\theta, \phi$. Then, $\mu=\frac{2}{r g}$. Since $g$ is only a function of $(t, r), \mu$ is spherically symmetric.

[1] S. Chandrasekhar, The Mathematical Theory of Black Holes, Clarendon Press, Oxford, (1992). 
[2] J.W. Bardeen, B. Carter and S.W. Hawking, Commun. Math. Phys. 31, 161 (1973).

[3] A. Ashtekar, C. Beetle and S. Fairhurst, Class. Quant. Grav.17, 253 (2000).

[4] R. M. Wald Phys. Rev. D48, 3427 (1993) and V. Iyer and R. M. Wald Phys. Rev. D50, 846 (1994).

[5] R. M. Wald and I. Racz Class. Quant. Grav. 13, 539 (1996).

[6] A. Ashtekar, C. Beetle and S. Fairhurst, Class. Quant. Grav. 16, L1 (1999).

[7] A. Ashtekar, C. Beetle, O. Dreyer, S. Fairhurst, B. Krishnan, J. Lewandowski and Jacek Wisniewski Phys. Rev. Lett. 85, 3564 (2000); A. Ashtekar, B. Krishnan and S. Fairhurst, Phys. Rev.D62, 104025 (2000).

[8] A. Ashtekar and B. Krishnan Liv. Rev. Rel. 710 (2004).

[9] R. M. Wald Liv. Rev. Rel. 4, 6 (2001).

[10] E. Gourgoulhon and J. L. Jaramillo Phys. Rept. 423, 159 (2006).

[11] A. Ashtekar, A. Corichi and K. Krasnov, Adv. Theo. Math. Phys. 3, 418 (1999).

[12] A. Ashtekar, J. C. Baez, A. Corichi and K. Krasnov, Phys. Rev. Lett. 80, 904 (1998).

[13] A. Ashtekar, J. C. Baez and K. Krasnov, Adv. Theo. Math. Phys. 4, 1 (2000); A. Ashtekar and A. Corichi, Class. Quant. Grav. 20, 4473 (2003); A. Ashtekar, J. Engle and C. van der Broeck, Class. Quant. Grav. 22, L27 (2004).

[14] A. Ashtekar, C. Beetle and J. Lewandowski, Phys. Rev. D64 044016 (2001); A. Ashtekar, C. Beetle and J. Lewandowski, Class. Quant. Grav.19, 1195 (2002); A. Ashtekar, A. Corichi and D. Sudarsky, Class. Quant. Grav. 20, 3413 (2003); A. Ashtekar, J. Engle, T. Pawlowski and C. van der Broeck, Class. Quant. Grav. 21, 2549 (2004).

[15] A. Chatterjee and A. Ghosh, Class. Quant. Grav. 23, 7521 (2006); A. Chatterjee AIP Conf. Proc. 939, 96 (2007).

[16] S. Holst, Phys. Rev. D56, (2002).

[17] A. Ashtekar Lectures on Non-perturbative Canonical Gravity (notes prepared in collaboration with R Tate), World Scientific, Singapore, (1991).

[18] A. Ashtekar and J. Lewandowski, Class. Quant. Grav., 21, R53R152 (2004)

19] C. Rovelli, T. Thiemann, Phys.Rev. D57 1009 (1998).

[20] J. D. Bekenstein, Phys. Rev. D7, 2333 (1973).

[21] S. W. Hawking, Commun. Math. Phys. 43, 199 (1975).

[22] B. de Wit B Proc. Symp. in Honor of Julius Wess on the Occasion of his 70th Birthday, (Munich, Germany) Fortschr. Phys. 54183 (2006)

[23] A. Sen J. High Energy Phys. 05059 (2005); J. High Energy Phys. 03008 (2006).

[24] A. Ghosh and P. Mitra Phys. Rev. Lett. 781858 (1997); G. L. Cardoso, B. de Wit and T. Mohaupt Fortschr. Phys. 48 49 (2000); K. Kiefer and J. Louko Ann. Phys. 8 67, (1999).

[25] R. Penrose and E. T. Newman J. Math. Phys. 1858 (1977).

[26] J. Stewart Advanced General Relativity Cambridge Univ Press, Cambridge (1990).

[27] L. Fatibene, M. Ferraris, M. Francaviglia, G. Pacchiella arXiv 0808.3845 [gr-qc].

[28] S. Carroll, M. Johnson, L. Randall arXiv 0901.0931 [hep-th]. 